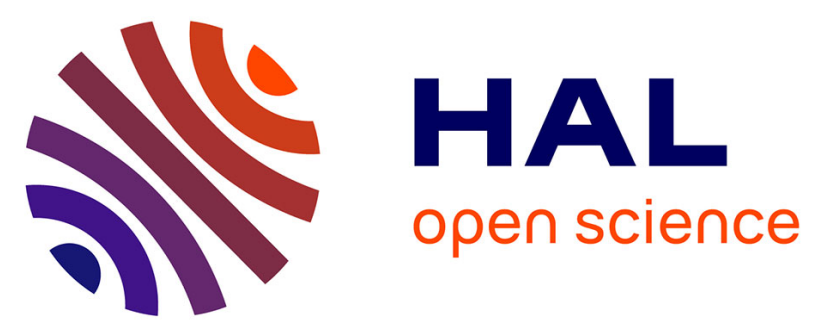

\title{
Intervention basée sur la pleine conscience auprès de patients souffrant d'obésité et de trouble de binge eating : résultats préliminaires de l'essai contrôlé randomisé MindOb
}

Alexis Ruffault, Sébastien Czernichow, Kàtia Lurbe I Puerto, Jean Fournier, Claire Carette, Cécile Flahault

\section{To cite this version:}

Alexis Ruffault, Sébastien Czernichow, Kàtia Lurbe I Puerto, Jean Fournier, Claire Carette, et al.. Intervention basée sur la pleine conscience auprès de patients souffrant d'obésité et de trouble de binge eating : résultats préliminaires de l'essai contrôlé randomisé MindOb. Journal de Thérapie Comportementale et Cognitive, 2019, 29 (1), pp.4-24. 10.1016/j.jtcc.2018.09.001 . hal-03032217

\section{HAL Id: hal-03032217 https: / hal-insep.archives-ouvertes.fr/hal-03032217}

Submitted on 22 Oct 2021

HAL is a multi-disciplinary open access archive for the deposit and dissemination of scientific research documents, whether they are published or not. The documents may come from teaching and research institutions in France or abroad, or from public or private research centers.
L'archive ouverte pluridisciplinaire HAL, est destinée au dépôt et à la diffusion de documents scientifiques de niveau recherche, publiés ou non, émanant des établissements d'enseignement et de recherche français ou étrangers, des laboratoires publics ou privés.

\section{(ㅇ)(1) $\$$}

Distributed under a Creative Commons Attribution - NonCommerciall 4.0 International 
Intervention basée sur la pleine conscience auprès de patients souffrant d'obésité et de trouble de binge eating : Résultats préliminaires de l'essai contrôlé randomisé MindOb

Mindfulness-based intervention among patients with obesity and binge eating disorder:

Preliminary results of the MindOb randomized controlled trial

Alexis Ruffault ${ }^{\mathrm{a}, \mathrm{b}, \mathrm{c},{ }^{*}, \text { Sébastien Czernichow }}{ }^{\mathrm{d}, \mathrm{e}, \mathrm{f}}$, Kàtia Lurbe i Puerto ${ }^{\mathrm{d}}$, Jean F. Fournier ${ }^{\mathrm{g}}$, Claire Carette $^{\mathrm{d}, \mathrm{e}}$, Cécile Flahault ${ }^{\mathrm{c}}$

${ }^{\mathrm{b}}$ Laboratoire Sport, Expertise et Performance (EA 7370), Unité Recherche, Institut National du Sport, de l'Expertise et de la Performance (INSEP), 11 avenue du Tremblay, 75012 Paris, France

${ }^{\mathrm{b}}$ Département de Psychologie de la Santé, Unité de Recherche interfacultaire Santé et Société (URiSS), Université de Liège, 30-32 rue de l'Aunaie, 4000 Liège, Belgique

${ }^{\mathrm{c}}$ Laboratoire de Psychopathologie et Processus de Santé (EA 4057), Institut de Psychologie, Université Paris Descartes, Sorbonne Paris Cité, 71 avenue Edouard Vaillant, 92100

Boulogne-Billancourt, France

${ }^{\mathrm{d}}$ Service de Nutrition, Hôpital Européen Georges-Pompidou, Assistance Publique-Hôpitaux de Paris (AP-HP), 20 rue Leblanc, 75015 Paris

${ }^{e}$ Faculté de Médecine, Université Paris Descartes, Sorbonne Paris Cité, 12 rue de l'École de Médecine, 75006 Paris, France

${ }^{\mathrm{f}}$ INSERM UMS 011, Cohortes épidémiologiques en population, 16 avenue Paul-Vaillant Couturier, 94800 Villejuif, France

${ }^{\mathrm{g}}$ UFR STAPS, Université Paris Nanterre, 200 avenue de la République, 92000 Nanterre, France

*Correspondant :

alexis.ruffault@insep.fr

Titre court: Pleine conscience et binge eating

Word count: 8641

\section{Remerciements}

Les auteurs souhaitent remercier la Fondation Nestlé France pour son aide financière, l'Unité de Recherche Clinique de l'Hôpital Ambroise Paré (AP-HP), et l'Unité de Recherche Clinique de l'Hôpital Européen Georges-Pompidou (AP-HP) pour leur aide dans les démarches technico-réglementaires. 


\section{PLEINE CONSCIENCE ET BINGE EATING}

1 Intervention basée sur la pleine conscience auprès de patients souffrant d'obésité et de trouble de binge eating : Résultats préliminaires de l'essai contrôlé randomisé MindOb

\section{Résumé}

5 La pleine conscience pourrait permettre de réduire l'impulsivité alimentaire et augmenter la

6 motivation à pratiquer des activités physiques, à condition d'être pratiquée quotidiennement

7 par des individus souffrant d'obésité et de trouble de binge eating. L'objectif est de tester

8 l'efficacité d'une intervention à distance basée sur la pleine conscience auprès de patients

9 obèses souffrant de trouble de binge eating et observer l'adhésion quotidienne mesurée de

10 manière objective.

11 Seize patients obèses présentant un trouble de binge eating ont été randomisés dans trois

12 groupes : pleine conscience, méditation factice, liste d'attente. Les deux interventions testées

13 consistaient en l'écoute de pistes audio sur internet, tous les jours pendant 12 mois. Le poids,

14 les processus alimentaires, les apports énergétiques, la motivation à l'activité physique, la

15 dépense énergétique, la détresse psychologique et les capacités de pleine conscience ont été 16 mesurés à l'inclusion, un mois, six mois et 12 mois.

17 Parmi les 16 patients inclus (dont sept abandons ou perdus de vue), aucun n'a suivi entièrement l'intervention ni pendant le premier mois (24,76\% d'audio écouté en moyenne), ni après la première visite de suivi (9,80\% d'audio écouté en moyenne).

Il semble donc difficile de tester l'efficacité d'une intervention à distance sans mettre en place

21 des techniques ayant pour objectif d'améliorer l'adhésion des participants.

22 Mots-clés : pleine conscience ; obésité ; trouble des accès hyperphagiques ; activité physique ; adhésion 


\section{PLEINE CONSCIENCE ET BINGE EATING}

Mindfulness-based intervention among patients with obesity and binge eating disorder: Preliminary results of the MindOb randomized controlled trial

(1)

\section{Abstract}

Introduction: Mindfulness is the ability to voluntarily focus on the present moment without judgment. Several systematic reviews and meta-analyses have demonstrated the effectiveness of mindfulness-based interventions among individuals with obesity, mainly on the reduction of binge episodes and related factors such as impulsive and emotional eating. Therefore, mindfulness could decrease impulsive eating and increase motivation toward physical activity. Furthermore, daily online mindfulness training for individuals diagnosed with binge eating disorder who benefit from usual care in a clinical nutrition department could be more adapted than standardized weekly group interventions.

Objective: To test the effectiveness of an online mindfulness-based intervention among patients with obesity and binge eating disorder, and to objectively observe measured daily adherence to the intervention.

Method: Sixteen patients with obesity and binge eating disorder (based on DSM5 criteria) were randomized to one of three of the following groups: mindfulness training $(n=5)$, sham meditation $(n=8)$, waitlist $(n=3)$. Both interventions consisted of online 10-minute daily audio sessions lasting for 12 months in addition to usual care in nutrition. Weight, eating processes (Three-Factor Eating Questionnaire R18), energy intake (7-day dietary recall), motivation toward physical activity (Behavioral Regulation Toward Exercise Questionnaire 2), energy expenditure (International Physical Activity Questionnaire and a 7-day pedometer log), psychological distress (Hospital Anxiety and Depression Scale), and mindfulness skills (Mindful Attention Awareness Scale and Daily Mindful Responding Scale over 7 days) were measured at baseline, one month, six months, and 12 months. An objective measure of 


\section{PLEINE CONSCIENCE ET BINGE EATING}

50

51

52

53

54

55

56

57

58

59

60

61

62

63

64

65

66

67

68

69

70

participants' adherence to both interventions was provided by the hosting server for each individual. Data was analyzed using the Reliable Change Index formulas to estimate the effect of the intervention on each participant on an individual change basis.

Results: From 47 patients potentially eligible for the MindOb study, 28 were screened for eligibility and 16 were randomized ( 10 women; mean age 40 years old \pm 14.37 ; mean BMI $\left.36.93 \mathrm{~kg} / \mathrm{m}^{2} \pm 4.24\right)$. The main result of the case series remains in the number of drop-outs $(n=4)$, and lost patients $(n=3)$. Among the 16 participants, none attended $100 \%$ of the online sessions neither during the first month $(24.76 \%$ of the sessions) nor after the first visit $(9.80 \%$ of the sessions). A majority of participants from the sham meditation group showed a decrease in impulsive and emotional eating, and an increase in extrinsic motivation for physical activity after one month of intervention. However, no participant in the intervention groups showed clinically significant change on mindfulness scores or increased scores of psychological distress after one month of intervention.

Conclusion: The results highlight the challenges in testing the effectiveness of online interventions among patients with obesity and binge eating disorder. Additional techniques (e.g., action planning) could increase adherence to such interventions. Furthermore, increasing the sample size and analyzing the 6- and 12-month data could result in an increase of statistical power as well as increased possibility to conduct group comparison analyses instead of an estimate of individual change.

Keywords: mindfulness; obesity; binge eating; physical activity; adherence 


\section{PLEINE CONSCIENCE ET BINGE EATING}

\section{Introduction}

En France en 2016, on estimait à 15,8\% d'hommes et 15,6\% de femmes souffrant d'obésité [1], c'est-à-dire présentant un indice de masse corporelle (IMC) supérieur ou égal à $30 \mathrm{~kg} / \mathrm{m}^{2}$. Ces chiffres sont proches des estimations mondiales [2] et mettent en évidence la nécessité d'agir sur les conduites alimentaires et les comportements actifs (c-à-d. les comportements directement impliqués dans la prise et la perte de poids) des individus souffrant d'obésité dans une prise en charge pluridisciplinaire [3,4]. Cependant, les techniques de prise en charge comportementales et médicales actuelles sont souvent mises en échec [5], et les thérapies cognitives et comportementales (TCC) pourraient permettre d'aider les patients à mieux gérer leurs apports alimentaires ainsi que leur dépense énergétique au quotidien. Plus précisément, les interventions basées sur la pleine conscience ont fait l'objet de nombreuses études au cours des dix dernières années auprès d'individus obèses ou en surpoids (IMC $\geq 25 \mathrm{~kg} / \mathrm{m}^{2}$ ), synthétisées dans plusieurs revues systématiques et méta-analyses principalement focalisées sur la prise en charge du trouble de binge eating [6-11]. En effet, le trouble de binge eating (ou accès hyperphagiques) est caractérisé par des épisodes hyperphagiques récurrents impliquant un sentiment de manque de contrôle (c-à-d. impulsivité) sur la prise alimentaire [12], et ce sans conduites compensatoires ayant pour objectif de contrôler le poids (p.ex. : vomissements). Selon les données épidémiologiques, ce trouble toucherait $1 \%$ de la population générale et 32,8 à $41,7 \%$ des individus souffrant d'obésité [13]. De plus, une étude expérimentale a montré que les individus en surpoids et obèses ont tendance à être plus impulsifs et à manger de plus grandes quantités de nourriture disponible après induction d'une émotion négative comparativement aux individus de poids normal [14]. Ainsi, les épisodes alimentaires sous-tendus par un manque de contrôle (c-à-d. impulsivité), comme les épisodes de binge eating, ont tendance à être amorcés par des états émotionnels négatifs [15]. 


\section{PLEINE CONSCIENCE ET BINGE EATING}

La pleine conscience désigne à la fois une capacité pour laquelle chacun est plus ou moins performant [16] et un courant psychothérapeutique (troisième vague des TCC) pour lequel les interventions ont pour objectif d'améliorer les capacités de pleine conscience des participants [17]. La pleine conscience est définie comme l'orientation volontaire de l'attention sur le moment présent, et ce, sans porter de jugement sur les pensées, émotions, sensations qui peuvent y survenir [18]. Il existe, dans la littérature, de nombreux programmes intégrant des séances de pleine conscience. Toutefois, ces programmes, bien que différents dans leur design, ont pour objectif commun d'augmenter les capacités de pleine conscience des individus qui y participent.

Dans le traitement du binge eating, les auteurs avancent qu'au lieu de chercher à changer les pensées et émotions négatives des patients qui utilisent l'alimentation comme réponse à la détresse psychologique, le but de la thérapie devrait être d'orienter les patients vers des stratégies de régulation des émotions plus adaptatives, et ce à l'aide des techniques de pleine conscience [19]. Dans la littérature, les mécanismes impliqués dans l'efficacité de la pleine conscience sur la réduction des symptômes de troubles alimentaires ont pu être mis en évidence. Il a ainsi été suggéré que la conscience intéroceptive, et plus particulièrement la non-acceptation des variations émotionnelles, seraient des mécanismes impliqués dans la relation entre pleine conscience et symptômes de troubles alimentaires [20] ; et, auprès de patients souffrant de troubles alimentaires, les mêmes auteurs ont montré que l'impulsivité était associée à des déficits de régulation des émotions, de faibles capacités de pleine conscience, et des déficits de conscience intéroceptive [20]. Par ailleurs, la pleine conscience pourrait réduire l'impulsivité alimentaire en permettant aux individus de se décentrer de leurs pensées automatiques et d'accepter les états émotionnels aversifs qui prédisent les épisodes de binge eating, comme le démontrent deux études testant des interventions brèves [21,22]. 


\section{PLEINE CONSCIENCE ET BINGE EATING}

D'autre part, les capacités de pleine conscience ont montré un effet modérateur sur la

122

123

124

125 relation entre la motivation intrinsèque à pratiquer une activité physique et le niveau d'activité physique dans la population générale [23]. Ces résultats suggèrent que les individus présentant de meilleures capacités de pleine conscience internalisent leur motivation ; c'est-àdire qu'ils pratiquent pour leur plaisir personnel plutôt que pour des raisons extérieures (p.ex. : pour faire plaisir à leur médecin). D'autre part, les individus présentant de meilleures capacités de pleine conscience ont tendance à agir en cohérence avec leurs intentions de changer [24], et cette relation serait plus forte pour les individus qui n'ont pas intégré l'activité physique dans leurs habitudes de vie (c-à-d. les individus sédentaires). De plus, un essai contrôlé randomisé a ainsi testé l'impact de la pleine conscience sur l'augmentation de l'activité physique [25] : un groupe contrôle recevait des séances d'éducation relatives aux bénéfices de la pratique physique, l'autre groupe suivait une intervention basée sur la pleine conscience dont le but était d'être plus actif. Les participants du groupe pleine conscience se sont rendus plus souvent au centre sportif que les participants du groupe contrôle pendant les cinq semaines d'intervention. Ces résultats permettent de conclure à l'efficacité de la pleine conscience pour la promotion de l'activité physique à court terme. Auprès d'individus en surpoids ou obèses, la méta-analyse de Ruffault et al. [10] a montré une taille d'effet modérée et significative sur l'amélioration du niveau d'activité physique des participants suivant une intervention basée sur la pleine conscience en comparaison des participants des groupes contrôles, suggérant ainsi que la pleine conscience pourrait favoriser l'adoption de comportements actifs.

La méta-analyse de Ruffault et al. [10] a mis en évidence les biais méthodologiques, l'efficacité globale, mais aussi l'efficacité relative à certaines caractéristiques méthodologiques des interventions basées sur la pleine conscience auprès d'individus en surpoids ou obèses dans des essais contrôlés randomisés. Les résultats de cette recension de la 


\section{PLEINE CONSCIENCE ET BINGE EATING}

146 littérature ont montré que les méthodes de recrutement des participants sont à haut risque de

147 biais puisque les participants n'étaient pas contactés de manière systématique, mais devaient

148 contacter un investigateur s'ils étaient intéressés par l'intervention proposée, ce qui implique

149 que les participants étaient déjà intéressés par les interventions basées sur la pleine

150 conscience. D'autre part, les études inclues dans la méta-analyse ont montré des tailles d'effet

151 larges des interventions testées sur la réduction du binge eating et de l'impulsivité alimentaire,

152 des tailles d'effets modérées sur l'augmentation du niveau d'activité physique, et une absence

153 de résultat sur la réduction du poids. Ainsi, des analyses de méta-régression ont permis de

154 mettre en évidence que les interventions ciblant les individus souffrant de trouble de binge

155 eating ainsi que les interventions les plus longues étaient plus efficaces. Ainsi, dans les essais

156 contrôlés randomisés testant l'efficacité d'une intervention basée sur la pleine conscience

157 devraient adopter une méthode de recrutement plus systématique, ciblée sur les individus à

158 risque, et des interventions d'une durée de plus de trois mois [10].

159 Le but de l'étude MindOb est d'évaluer l'efficacité d'une intervention quotidienne à

160 distance basée sur la pleine conscience auprès de patients obèses atteints de trouble de binge

161 eating. L'intervention testée a pour but d'améliorer les capacités de pleine conscience des

162 patients obèses afin de les aider dans la gestion de leur impulsivité alimentaire et de leur

163 motivation à pratiquer des activités physiques. L'intervention testée est complémentaire au

164 suivi médical et paramédical proposé aux patients obèses pris en charge en service de

165 nutrition. Le design à distance a été choisi afin de ne pas obliger les patients à venir plus

166 souvent à l'hôpital, et pour éviter d'infliger une charge de travail supplémentaire à l'équipe

167 médicale. La pleine conscience, qui est un entraînement à prendre conscience et à se décentrer

168 des pensées et émotions automatiques d'une part, et comme modérateur du changement de

169 comportement d'autre part, pourrait permettre de réduire l'impulsivité alimentaire de patients

170 obèses atteints de trouble de binge eating (hypothèse $1 ; \mathrm{H} 1$ ) et d'améliorer leur motivation 


\section{PLEINE CONSCIENCE ET BINGE EATING}

171 intrinsèque à pratiquer des activités physique (hypothèse 2 ; H2), respectivement. Les

172 résultats préliminaires après six mois de participation sont présentés dans cet article sous

173 forme de série de cas cliniques, dont l'objectif est d'observer l'adhésion des participants à

174 l'intervention quotidienne. Le protocole détaillé et complet de l'étude MindOb a été publié par 175 ailleurs [26].

Méthode

178

Design de l'étude.

MindOb est une étude psychologique interventionnelle ouverte, prospective et contrôlée randomisée en trois groupes. Une randomisation préalable à l'inclusion permet d'assurer un contrôle des biais de confusion et de sélection entre les trois groupes. Les patients du groupe "pleine conscience" (groupe intervention, GI) ont eu une prise en charge usuelle de leur obésité et ont bénéficié d'une intervention basée sur la pleine conscience pendant douze mois. Les patients du groupe "méditation factice" (groupe intervention contrôle, GIC) ont eu une prise en usuelle de leur obésité, et ont bénéficié d'une intervention contrôle qui consiste en de la méditation non guidée pendant douze mois [27]. Les patients du groupe contrôle (GC) ont eu une prise en charge usuelle de leur obésité. Pour ces trois groupes, la prise en usuelle de l'obésité dans le service de nutrition se fait en même temps que leur participation à l'étude, soit au moins douze mois (certains étaient suivis avant l'étude et seront encore suivis dans le service après leur participation à l'étude). Les deux groupes contrôles ont permis de vérifier si les changements observés chez les patients étaient bien le résultat de l'intervention testée (c-àd. pleine conscience), et pas seulement de la simple proposition d'une intervention. La randomisation a été stratifiée sur le genre (homme ou femme), l'IMC (IMC $<40$ ou IMC $\geq 40$ ) et l'âge (âge $<46$ ou âge $\geq 46$ ). La valeur seuil de l'IMC a été choisie pour sa significativité clinique puisqu'un IMC supérieur à $40 \mathrm{~kg} / \mathrm{m}^{2}$ représente le critère de 


\section{PLEINE CONSCIENCE ET BINGE EATING}

196

197

198

199

200

201

202

203

204

205

206

207

208

209

210

classification du poids en obésité morbide. La valeur seuil de l'âge représente l'âge moyen des patients du service de nutrition pour l'année 2015. Cette étude de douze mois a examiné les effets de l'intervention testée sur des variables biomédicales, comportementales et psychologiques mesurées à l'inclusion, un mois, six mois et douze mois. Les participants ont tous donné leur consentement écrit avant de participer à l'étude et leur anonymat lors des analyses a été préservé. Un avis éthique favorable a été obtenu par le Comité d'évaluation éthique des projets de recherche en santé (CERES) ainsi que par le Comité de Protection des Personnes Ile-de-France II (CPP, numéro ID-RCB : 2015-A01370-49). D'autre part, une autorisation légale a été obtenue auprès de l'Agence Nationale de Sécurité du Médicament et des produits de santé (ANSM) et par le Comité Consultatif sur le Traitement de l'Information en matière de Recherche dans le domaine de la Santé (CCTIRS). Aussi, une déclaration à la Commission Nationale de l'Informatique et des Libertés (CNIL) a été effectuée. L'étude a également été enregistrée dans le registre international des recherches biomédicales

ClinicalTrials.gov (numéro : NCT02571387).

\section{Participants et procédure.}

Les participants sont recrutés sur une période de douze mois dans le service de l'Hôpital Ambroise-Paré et de l'Hôpital Européen Georges-Pompidou (HEGP) de l'Assistance Publique - Hôpitaux de Paris (AP - HP) par deux investigateurs psychologues au cours d'entretiens semi-structurés, systématiquement après leur visite médicale dans l'un des deux services. Les critères d'inclusion et de non-inclusion complets sont disponibles en matériel supplémentaire (matériel supplémentaire 1). Les critères diagnostiques du DSM5 [12] pour le trouble de binge eating ont été vérifiés par les deux investigateurs psychologues de l'étude au cours de la visite d'inclusion.

L'étude MindOb adoptait une procédure de double-aveugle partiel, de sorte que les patients randomisés dans un des deux groupes intervention (c-à-d. GI ou GIC), ainsi que 


\section{PLEINE CONSCIENCE ET BINGE EATING}

221 l'investigateur, ne puissent pas savoir de quelle intervention il s'agit. En revanche,

222 l'investigateur avait connaissance de l'appartenance au groupe sans intervention (c-à-d. GC)

223 puisque la procédure de participation était différente entre ce groupe et les groupes

224 intervention. Le numéro d'inclusion de chaque participant ainsi que l'allocation à un groupe

225 intervention ou contrôle a été générée aléatoirement par un programme informatique

226 (plateforme ClinShare par MyGoodLife). Les Attachés de Recherche Clinique (ARC) se sont

227 assurés que la procédure de randomisation ait bien été respectée. Une fois le participant

228 randomisé dans un groupe (GC ou GI/GIC), l'investigateur l'informait de tous les détails de sa

229 participation.

À l'inclusion (V0), un mois (V1), six mois (V6) et douze mois (V12), les patients

231 devaient prendre rendez-vous avec un investigateur à l'hôpital. Ces visites avaient pour but

232 principal de garder les participants motivés et de répondre aux questions concernant leur

233 participation. Avant chaque visite, les participants devaient répondre aux questionnaires en

234 ligne mesurant leur niveau d'activité physique déclaré, les processus psychologiques de leur

235 alimentation, leur motivation à pratiquer des activités physiques, leur anxiété, leur dépression,

236 et leurs capacités de pleine conscience. Pendant sept jours consécutifs à l'inclusion, puis à six

237 mois et douze mois, les participants devaient reporter sur papier leur niveau d'activité

238 physique mesuré objectivement et leurs capacités de pleine conscience au quotidien, et

239 remplir une enquête alimentaire en ligne. Au cours de la visite d'inclusion, de six mois et de

240 douze mois, les participants se présentaient pour être pesés par des Infirmier(ère)s

241 Diplômé(e)s d'État (IDE).

242 À l'inclusion, les investigateurs ont fourni aux participants : une lettre d'information

243 avec un formulaire de consentement signé par l'investigateur principal ainsi qu'un lien pour

244 s'enregistrer sur la plate-forme d'intervention avec un code à 12 chiffres. À la fin de la visite

245 d'inclusion, les participants recevaient les dates de leurs prochains rendez-vous, différents 


\section{PLEINE CONSCIENCE ET BINGE EATING}

246 plateformes en ligne de l'étude, ainsi qu'un podomètre (Geonaute ONWalk 100).

L'intervention a été présentée comme un entraînement psychologique complémentaire à

249 leur suivi en nutrition aux participants des groupes GI et GIC, et comme une étude visant à

mieux comprendre le vécu psychologique d'individus atteints de trouble de binge eating

auprès des patients du groupe GC. À la fin de l'étude, les participants du groupe GC se sont

vus proposer l'opportunité de bénéficier d'une des deux interventions (c-à-d. pleine conscience

ou méditative factice). De même, les participants du groupe GIC se sont vus proposer

l'opportunité de bénéficier de l'intervention pleine conscience. Toutefois, aucune mesure n'a

été effectuée après les 12 mois de participation de chaque patient ; l'efficacité de l'intervention dont ils ont bénéficié après la fin de l'étude n'a pas été évaluée.

\section{Intervention.}

Les séances de pleine conscience du groupe GI suivent les directives des principaux

259 programmes d'interventions basées sur la pleine conscience comme le Mindfulness-Based

Stress Reduction [18], le Mindfulness-Based Cognitive Therapy [28], ou encore l'Acceptance

and Commitment Therapy [29], en ce qui concerne la construction des séances à écouter en

262 autonomie dans ces programmes. Les participants des trois groupes ont bénéficié du même

263 suivi usuel de leur obésité. Ce suivi usuel en nutrition inclut des séances d'éducation

264 thérapeutique pluridisciplinaires en groupe (alimentation, activité physique, vécu

265 psychologique, prise en charge médicale) ainsi que des consultations individuelles avec des

266 médecins nutritionnistes, des diététicien(ne)s, des éducateur(rice)s médico-sportif(ve)s et des

267 psychologues. Des informations personnalisées concernant la balance énergétique, les

268 comorbidités médicales, les régimes alimentaires, l'activité physique, la détresse

269 psychologiques ou encore les troubles alimentaires sont données aux patients du service.

270 Cette prise en charge usuelle de l'obésité suit les recommandations en vigueur de la Haute 


\section{PLEINE CONSCIENCE ET BINGE EATING}

Autorité de Santé [3].

Les séances étaient disponibles au format audio sur une page internet personnelle mise à disposition de chaque participant des groupes GI et GIC (plateforme 31k). Ainsi, tous les jours pendant dix minutes, les patients devaient se rendre sur leur page internet pour écouter leur séance. Une phase planifiée de l'écoute des séances était effectuée pendant les quatre premières semaines d'intervention, selon un planning imposé par l'investigateur. Ainsi, tous les sept jours pendant la phase planifiée, le contenu des séances était modifié à distance par un investigateur pour chaque patient inclus. À la suite du premier mois d'intervention planifiée, les patients avaient pour consigne de continuer d'écouter une séance de pleine conscience de 10 minutes tous les jours. Au cours de cette phase autonome d'intervention, les patients étaient libres dans le choix des séances à écouter. La procédure d'intervention pour chaque groupe de l'étude est illustrée dans la figure 1. Pendant les deux phases d'intervention (c-à-d. planifiée et autonome), un audio supplémentaire d'une durée de trois minutes était disponible et permettait aux patients de comprendre le but de l'intervention. Cet audio supplémentaire expliquait notamment aux participants que le but de l'intervention était d'apprendre à se concentrer sur l'instant présent en étant pleinement conscient des pensées, émotions et sensations pouvant arriver pendant la séance afin d'améliorer la qualité de leur contrôle personnel et d'adopter un mode de vie sain.

\section{METTRE ICI FIGURE 1}

L'intervention testée auprès des participants du groupe GI (c-à-d. pleine conscience) comportait quatre types de séances. Une séance d'orientation de l'attention sur la respiration au cours de laquelle le patient devait se tenir assis, les yeux fermés, dans une position confortable et dans un lieu calme. Les consignes orientaient l'attention du patient vers sa respiration, de manière à ce qu'il prenne conscience des changements induits par les mouvements respiratoires, tout en prenant conscience des pensées qui pouvaient arriver et 


\section{PLEINE CONSCIENCE ET BINGE EATING}

296

297

298

299

300

301

302

303

304

305

306

307

308

perturber l'exercice. Un fond sonore de bruits de forêt était audible sans être gênant derrière les consignes données dans cette séance. Une séance d'orientation de l'attention sur la posture au cours de laquelle le patient devait se tenir assis ou debout (selon son propre choix), les yeux fermés, dans une position confortable et dans un lieu calme. L'attention du patient était orientée vers ses appuis au support (c-à-d. la chaise s'il est assis, le sol s'il est debout), ainsi que sur son équilibre postural tout en prenant conscience des pensées qui pouvaient arriver et perturber l'exercice. Un fond sonore de bruits de la nuit était audible sans être gênant derrière les consignes données dans cette séance. Une séance d'entraînement à l'acceptation des pensées et émotions au cours de laquelle le patient devait se tenir assis, les yeux fermés, dans une position confortable et dans un lieu calme. L'attention du patient était orientée vers les pensées et émotions qui lui traversaient l'esprit dans l'instant présent. Ainsi, lorsqu'une pensée ou une émotion arrivait au patient, le patient devait en prendre conscience et la laisser passer. Un fond sonore de bruits de plage était audible sans être gênant derrière les consignes données dans cette séance. Une séance d'orientation de l'attention sur un mouvement simple au cours de laquelle le patient devait se tenir debout, les yeux fermés ou ouverts, dans un lieu calme. Chaque minute, le patient était invité à faire une flexion avec ses deux jambes (avec un appui au mur pour éviter les chutes si nécessaire) et de prendre conscience des sensations et des changements musculaires et posturaux que ce mouvement entraînait. Le patient devait également prendre conscience de la difficulté (ou de la facilité) qu'il éprouvait à réaliser ce mouvement. Un fond sonore de bruits de rivière était audible sans être gênant derrière les consignes données dans cette séance. Aucune séance n'avait pour objectif de travailler spécifiquement sur l'alimentation (p. ex. : faim, satiété), puisque l'intervention de l'étude MindOb avait pour objectif d'améliorer les capacités de pleine conscience des participants, quelle que soit la situation de la vie quotidienne. 


\section{PLEINE CONSCIENCE ET BINGE EATING}

321 bruits de la forêt, de la nuit, de la plage et de la rivière. Chaque séance de méditation factice

322

323

324

325 commençait par une consigne invitant les participant à méditer (sans donner aucune consigne supplémentaire qui pourrait les aider à "méditer") et se terminait par une annonce précisant que la séance était terminée. Ce type d'intervention contrôle est ce qui se rapproche le plus d'un placebo de la pleine conscience et a été introduit dans la littérature par Zeidan et al. [26]. Mesures effectuées.

Directement après la vérification des critères d'inclusion et la randomisation des patients, au cours de la visite V0 (c-à-d. inclusion), un investigateur procédait au remplissage du cahier d'observation électronique (eCRF) avec les patients sur la plate-forme ClinShare (MyGoodLife). L'eCRF comportait des catégories de questions à choix multiples telles que le niveau d'éducation, la catégorie socioprofessionnelle et socioéconomique, la situation familiale, la spiritualité, les informations médicales, les suivis psychologiques antérieurs, la connaissance préalable de la pleine conscience, l'aise avec les nouvelles technologies, les intentions de changement et la motivation à participer à l'étude. Ces questions ont été adaptées d'études sociologiques précédentes [30]. Pour chaque question, les participants avaient deux choix supplémentaires "je ne souhaite pas répondre" et "je ne sais pas répondre" afin d'éviter les réponses par défaut que certains patients pourraient donner et d'éviter les données manquantes liées à une réelle envie de ne pas répondre ou une incapacité à proposer une réponse.

Un(e) IDE aveugle au groupe dans lequel les participants étaient randomisés procédait aux mesures de la taille (en centimètres) et du poids (en kilogrammes) des patients. La taille était mesurée à l'inclusion, et le poids à l'inclusion, six mois (V6) et douze mois (V12). L'IMC a été calculé à partir de ces mesures suivant la formule $\mathrm{IMC}=$ poids $(\mathrm{kg}) /$ taille $\left(\mathrm{m}^{2}\right)$. Le Three-Factor Eating Questionnaire (TFEQ-R18) a été utilisé à V0, V1 (un mois), V6 et V12. Il s'agit un auto-questionnaire en 18 items qui mesure les processus cognitifs et 


\section{PLEINE CONSCIENCE ET BINGE EATING}

comportementaux des conduites alimentaires en trois facteurs [31] : l'impulsivité alimentaire (épisodes de perte de contrôle), la restriction cognitive (contrôle des apports alimentaires pour influencer le poids) et l'alimentation émotionnelle (hyperphagie lors d'épisodes thymiques dysphoriques). Les items 1 à 17 sont évalués sur une échelle de Likert allant de 1 à 4, et l'item 18 est évalué sur une échelle de Likert allant de 1 à 8 mais codé comme suit : 1-2 en 1, 3-4 en 2, 5-6 en 3, 7-8 en 4. La dernière étude de validation du TFEQ-R18 auprès d'individus obèses en Suède a montré une consistance interne ( $\alpha$ de Cronbach) pour chaque sous-échelle allant de 0,78 à $0,94[31,32]$.

À l'inclusion (V0), V6 et V12, les participants ont également été invités à remplir une enquête alimentaire en ligne pendant sept jours consécutifs sur la plateforme ClinShare (MyGoodLife). Ainsi, chaque jour de mesure les participants ont déclaré ce qu'ils ont mangé à chaque repas (c-à-d. petit déjeuner, déjeuner, diner) à l'aide d'une liste d'items et en indiquant la quantité absorbée de chaque item (c-à-d. aliment). Les données de deux jours aléatoires de semaine et d'un jour aléatoire de week-end ont été conservées pour l'analyse des données [33]. Un(e) diététicien(ne) aveugle au groupe dans lequel les participants étaient randomisés a vérifié les données pour identifier les données invalides (c-à-d. valeurs impossibles). Le Behavioral Regulation in Exercise Questionnaire (BREQ-2) a été utilisé à V0, V1, V6 et V12. Il s'agit d'un auto-questionnaire en 19 items mesurant la motivation à pratiquer des activités physiques en cinq sous-échelles [34] : l'amotivation (absence de motivation à changer de comportement), la régulation externe (engagement dans les activités physiques pour satisfaire une pression extérieure ou obtenir une récompense de l'extérieur), la régulation introjectée (internalisation des pressions extérieures pour éviter les émotions négatives ou renforcer les émotions positives qui y sont liées), la régulation identifiée (acceptation consciente de l'activité physique comme importante pour obtenir des récompenses autogénérée) et la régulation intrinsèque (engagement dans l'activité physique pour la satisfaction 


\section{PLEINE CONSCIENCE ET BINGE EATING}

371 inhérente qu'elle procure). Tous les items sont évalués sur une échelle de Likert en cinq points

372 allant de 0 (désaccord) à 4 (accord), et le score pour chacune des sous-échelles correspond à

373 la moyenne des items de chaque sous-échelle. L'étude de validation du BREQ-2 a montré une

374 consistance interne ( $\alpha$ de Cronbach) pour chaque sous-échelle allant de 0,73 à 0,86 [34].

375 Le niveau auto-reporté d'activité physique a été évalué avec la version courte de

376 l'International Physical Activity Questionnaire (IPAQ) à V0, V1, V6 et V12 [35]. L'IPAQ

377 fournit des informations concernant le temps que les participants passent à (a) pratiquer des

378 activités physiques intenses, (b) pratiquer des activités physiques d'intensité modérée, (c)

379 marcher et (d) rester assis, au cours de sept jours consécutifs. Les scores ont été calculés avec

380 un coefficient 8 pour les activités intenses, 4 pour les activités d'intensité modérée et 3,3 pour

381 la marche, puis traduits en dépense énergétique exprimée en Metabolic Equivalent Total

382 (MET) selon les formules : activités intenses (MET-min/semaine) $=8.0 \times$ activités intenses

383 (minutes) $\times$ activités intenses (jours); activités modérées $($ MET-min/week) $=4.0 \times$ activités

384 modérées (minutes) $\times$ activités modérées (jours) ; marche (MET-min/week) $=3.3 \times$ marche

385 (minutes) $\times$ marche (jours). Le niveau total d'activité physique était calculé suivant la formule

386 : activité physique totale $($ MET-min/semaine $)=$ activités intenses + activités modérées +

387 marche.

388 Le niveau d'activité physique était également mesuré de manière objective avec un

389 podomètre (Geonaute ONWalk 100). Il a été demandé aux participants de porter un

390 podomètre pendant sept jours consécutifs à l'inclusion, six mois (V6) et 12 mois (V12), et de

391 reporter la distance parcourue (en kilomètres) et le nombre de pas effectués chaque soir dans

392 leur carnet de participation. Le Geonaute ONWalk 100 est un senseur en trois dimensions

393 ayant pour fonction de mesurer la quantité de marche effectuée au quotidien. Les participants

394 pouvaient ainsi mettre le podomètre dans une de leurs poches, dans leur sac ou le clipser à

395 leur ceinture. Des piles supplémentaires ont été fournies aux participants pour qu'ils puissent 


\section{PLEINE CONSCIENCE ET BINGE EATING}

396 les changer en cas de besoin sur la période de 12 mois de participation à l'étude. Les

397 participants pouvaient utiliser leur podomètre en dehors des périodes de mesure, pour leur

398 utilisation personnelle, cependant ils devaient reporter sur leur carnet de participation le

399 nombre de jours additionnels pendant lesquels ils ont utilisé leur podomètre.

L'Hospital Anxiety and Depression Scale (HADS) a été utilsée à V0, V1, V6 et V12.

401 C'est une mesure auto-reportée de l'anxiété (sept items) et de la dépression (sept items)

402 validée auprès d'individus souffrant de maladies somatiques [36]. Les deux facteurs (c-à-d.

403 anxiété et dépression) sont évalués sur une échelle de Likert allant de 0 à 3 ; le score à chaque

404 facteur correspondant à la somme des sept items de chacun des deux facteurs. Dans la

405 littérature, l'HADS a montré une consistance interne ( $\alpha$ de Cronbach) de 0,83 en moyenne

406 pour l'anxiété et de 0,86 en moyenne pour la dépression [37].

407

Les capacités de pleine conscience étaient évaluées avec deux auto-questionnaires pour

vérifier si les effets de l'intervention étaient bien liés à l'amélioration des dispositions à être

pleinement conscient (c-à-d. mesurées avec la Mindful Attention Awareness Scale; MAAS) à

V0, V1, V6 et V12, et de la capacité à être pleinement conscient dans des situations du

quotidien en parallèle d'un entraînement à la pleine conscience (c-à-d. mesurée avec la Daily

Mindful Responding Scale; DMRS) à V0, V6 et V12. Ainsi, les caractéristiques

MAAS [16] montre que l'outil présente une consistance interne ( $\alpha$ de Cronbach) allant de 0,80

417 à 0,87 dans sept échantillons de participants différents. La DMRS [38] est composée de

418 quatre items évalués sur une échelle de Likert allant de 1 à 10, et dont le score correspond à la

419 moyenne des quatre items. Les participants ont répondu à la DMRS sur sept jours consécutifs

420 à l'aide de leur carnet de participation. La validation de l'outil auprès de participants à une 


\section{PLEINE CONSCIENCE ET BINGE EATING}

421 intervention basée sur la pleine conscience a montré une consistance interne ( $\alpha$ de Cronbach)

422 de $0,83[38]$.

423 L'adhésion à l'intervention a été enregistrée comme le quotient du nombre de séances

424 écoutées et du nombre de séances prescrites pour les groupes GI et GIC (c-à-d. bénéficiant

425 d'une intervention). Le nombre de séances écoutées correspond au temps passé sur la

426 plateforme $31 \mathrm{k}$ à écouter une séance, et ce, pour chaque participant. Cette mesure a été faite

427 de manière objective puisqu'il s'agissait des données de connexion des participants à la

428 plateforme d'intervention. D'autre part, lors des visites V1, V6 et V12, le discours des

429 participants au sujet de leur utilisation de la plateforme a été enregistré par les investigateurs

430 sous forme de catégories d'arguments concernant leurs croyances, leurs motivations à

431 continuer, les barrières à continuer, ainsi que les moyens qu'ils ont mis en place pour

432 continuer à écouter les séances (p.ex. : mise en place de rappels sur leur téléphone). Au cours

433 de ces trois visites, dans le but d'obtenir les éléments précédemment cités, les investigateurs

434 (c-à-d. psychologues) ont utilisés les principes de l'approche centrée sur la personne de

435 Rogers [38].

436 Analyse des données préliminaires.

437 Les résultats de cette étude sont présentés sous forme d'une série de cas contrôlés dans

438 cet article. Ainsi, les moyennes et écarts types à chaque temps de mesure pour chaque groupe

439 sont présentés. Étant donné la taille de l'échantillon $(n=16)$ et la répartition dans les groupes,

440 aucune comparaison de groupes n'est effectuée sur le plan statistique.

441 De plus, pour chaque outil de mesure, un indice fiable du changement (Reliable Change

442 Index; RCI) a été calculé comme score seuil, permettant de définir si les changements

443 observés dans le temps pour chaque participant sont bien le fait de sa participation à l'étude

444 plutôt qu'au hasard [40]. Cet indice a pour avantages d'être applicable à des échantillons de

445 petite taille et de permettre d'établir une norme au sein de l'échantillon en se basant sur la 


\section{PLEINE CONSCIENCE ET BINGE EATING}

446

447

dispersion des scores à l'inclusion [41]. L'équation prend en compte l'erreur standard et la consistance interne à l'inclusion pour l'ensemble de l'échantillon. Un changement pour un participant est considéré cliniquement significatif lorsque la différence de score dans le temps se situe en dehors de l'intervalle de confiance à $95 \%$ du RCI. Dans les cas où la consistance interne de l'outil de mesure était impossible à calculer (p.ex. : mesures de l'activité physique, de l'alimentation et du poids), le RCI ne pouvait pas être estimé. Pour ces mesures, la différence de score pour un individu entre l'inclusion et le suivi (c-à-d. un et six mois) devait se situer en dehors d'un intervalle de deux écarts types autour de la moyenne à l'inclusion (cà-d. entre $\mathrm{M}_{\mathrm{T} 0}-2 \mathrm{SD}_{\mathrm{T} 0}$ et $\mathrm{M}_{\mathrm{T} 0}+2 \mathrm{SD}_{\mathrm{T} 0}$ ) pour être considérée comme cliniquement significative.
Résultats

Description de la population et attrition.

Entre juillet 2016 et mars 2017, 47 patients ont été adressés par les médecins nutritionnistes pour une vérification des critères d'inclusion. Dix-neuf patients ont été perdus de vue au moment d'être contactés par l'investigateur (c-à-d. ils ne répondaient pas au téléphone ni aux mails malgré plusieurs tentatives) et n'ont donc pas pu être évalués pour les critères d'éligibilité. D'autre part, 12 patients évalués n'ont pas été inclus : cinq (quatre femmes, un homme) parce qu'ils ne souhaitaient pas participer à cette étude (trois refus de suivi à distance, deux pour contraintes professionnelles), quatre (deux femmes, deux hommes) parce qu'ils n'avaient pas de trouble de binge eating, une parce qu'elle présentait des bouffées délirantes aiguës fréquentes, une parce qu'elle présentait une obésité d'origine génétique, une parce qu'elle était enceinte. Enfin, 16 patients éligibles ont été inclus dans l'étude MindOb et randomisés. 


\section{PLEINE CONSCIENCE ET BINGE EATING}

471 de 16 patients inclus est composé de dix femmes et six hommes, âgés en moyenne de 40 ans

$472( \pm 14,37)$, avec un IMC moyen de $36,93 \mathrm{~kg} / \mathrm{m} 2( \pm 4,24)$. Quatre patients présentaient les cinq

473 symptômes du critère B de trouble de binge eating, six présentaient quatre symptômes sur

474 cinq, et six présentaient trois symptômes sur cinq. Onze participants ont bénéficié d'un suivi

475 psychologique par le passé, et six sont encore suivis actuellement. Treize participants ont

476 évalué leur intention de participer à une prise en charge psychothérapeutique expérimentale à

4776 ou 7 sur 7. Dix participants se disent accessibles à la spiritualité sous toutes ses formes (c-à-

478 d. qu'elle soit définie comme la relation avec un être supérieur, ou comme l'action de l'esprit).

479 Neuf participants pratiquent un sport en club ou en association sportive et onze se définissent

480 comme actifs au quotidien. Sept participants ont évalué leur intention de suivre les

481 recommandations en termes d'activité physique à 12, 13, ou 14 sur 14 (c-à-d. au moins 6 de

482 moyenne sur les deux items). De plus, cinq participants ont évalué leur intention de suivre les 483 recommandations diététiques à 12, 13, ou 14 sur 14 (c-à-d. au moins 6 de moyenne sur les 484 deux items). Enfin, dix participants ont évalué leur aise avec l'informatique à 6 ou 7 sur 7. METTRE ICI TABLEAU 1

Parmi les seize participants randomisés, une femme de 34 ans (P011) incluse dans la 487 condition contrôle active et un homme de 24 ans (P005) inclus dans le groupe expérimental ont été perdus de vue entre l'entretien d'inclusion et le premier temps de mesure. Un homme de 53 ans (P014) inclus dans la condition contrôle active a été perdu de vue après un mois de participation. Dans la condition expérimentale, une femme de 52 ans (P001) et une femme de

49132 ans (P010) ont retiré leur consentement après 32 et 8 jours, respectivement. Dans la

492 condition contrôle active, une femme de 34 ans (P008) a retiré son consentement après 23

493 jours et une femme de 58 ans (P007) a retiré son consentement après six mois de

494 participation. Ces sept patients font partie du groupe "abandons et perdus de vue" de l'analyse 495 des résultats. 


\section{PLEINE CONSCIENCE ET BINGE EATING}

Ainsi, trois participants de la condition contrôle inactive, quatre de la condition contrôle active et deux de la condition expérimentale ont rempli les mesures à l'inclusion et à un mois de suivi. Quatre participants (deux dans la condition contrôle inactive, un dans la condition contrôle active et un dans la condition expérimentale) ont également rempli les mesures à six mois de suivi. La figure 2 représente le flux de participants dans l'étude MindOb entre la vérification des critères d'inclusion et V6. Les scores des participants aux mesures effectuées à l'inclusion, à un mois et à six mois de suivi sont détaillés dans le tableau 2. Les données à un et six mois ne permettent pas d'établir de conclusion en raison du manque de données dans les groupes GI et GIC. C'est pourquoi les résultats ont été présentés de manière individuelle dans le tableau 3.

\section{METTRE ICI FIGURE 2}

\section{METTRE ICI TABLEAU 2}

\section{METTRE ICI TABLEAU 3}

\section{Conduites alimentaires.}

À l'inclusion, malgré les effectifs inégaux entre les trois groupes de patients $\left(\mathrm{n}_{\mathrm{GI}}=3\right.$; $\mathrm{n}_{\mathrm{GIC}}=6 ; \mathrm{n}_{\mathrm{GC}}=3$ ), certaines tendances sont observées sur les scores au TFEQ. En effet, les patients du groupe GI semblent présenter des scores moins élevés de restriction cognitive, d'impulsivité alimentaire et d'alimentation émotionnelle que les patients des groupes GIC et GI (voir tableau 2).

À un mois de participation, la patiente du groupe GI (P004) n'a pas montré d'évolution cliniquement significative des processus alimentaires. Cependant, à un mois de participation, quatre patients sur six du groupe GIC ont montré des améliorations cliniquement significatives de leurs processus alimentaires. En effet, la patiente P007 a présenté une augmentation de ses scores de restriction cognitive, les patientes P007 et P016 ont montré une diminution de leurs scores d'impulsivité alimentaire, et les patients P014, P015 et P016 ont 


\section{PLEINE CONSCIENCE ET BINGE EATING}

521 diminué leurs scores d'alimentation émotionnelle. En opposition, un patient sur deux du

522 groupe GC (P002) a diminué sa restriction cognitive et augmenté son alimentation

523 émotionnelle après un mois de participation (voir tableau 3).

524 À six mois de participation, la patiente du groupe GI (P004) et la patiente du groupe GC

525 (P013) n'ont montré d'évolution significative ni de leurs processus alimentaires, ni de leur

526 consommation journalière, ni de leur poids. Toutefois, le patient du groupe GIC (P006) a

527 réduit de manière cliniquement significative son impulsivité alimentaire et son alimentation

528 émotionnelle (voir tableau 3). Ces résultats suggèrent qu'une intervention factice basée sur la

529 pleine conscience pourrait permettre d'observer des effets bénéfiques sur les conduites

530 alimentaires de patients obèses atteints de trouble de binge eating dès un mois de

531 participation, maintenus jusqu'à six mois.

532 Activité physique.

533 À l'inclusion, malgré les effectifs inégaux entre les trois groupes de patients $\left(\mathrm{n}_{\mathrm{GI}}=3\right.$;

$534 \mathrm{n}_{\mathrm{GIC}}=6 ; \mathrm{n}_{\mathrm{GC}}=3$ ), certaines tendances sont observées sur les critères de jugement liés à

535 l'activité physique. En effet, les patients du groupe GC semblent plus amotivés et moins

536 intrinsèquement motivés à pratiquer des activités physiques que les patients des groupes GI et

537 GIC. De plus, les patients du groupe GI semblent avoir une dépense énergétique liée à

538 l'activité physique (c-à-d. score à l'IPAQ) moins élevée que les patients des groupes GIC et

539 GC, respectivement. Toutefois, lorsque la mesure du niveau d'activité physique se fait par

540 podomètre avec comme critère le nombre de pas effectués, les patients du groupe GC

541 semblent être moins actifs que les patients des groupes GI et GIC (voir tableau 2). Ces

542 résultats suggèrent que les patients du groupe GC pratiquent des activités physiques en dehors

543 de leur activité quotidienne de marche, malgré un profil motivationnel moins favorable.

544 À un mois de participation, la patiente du groupe GI ne semble pas présenter d'évolution

545 cliniquement significative sur les mesures liées à l'activité physique. De plus, trois patients du 


\section{PLEINE CONSCIENCE ET BINGE EATING}

546

547

548

549

550

551

552

553

554

555

556

557

558

559

560

561

562

563

564

565

566

567

568

569

570

groupe GIC sur six présentent des changements cliniquement significatifs de leurs

motivations à pratiquer des activités physiques. En effet, le patient P014 présente une

augmentation de l'amotivation, les patients P006 et P015 montrent une augmentation de leur

motivation extrinsèque, et les patients $\mathrm{P} 014$ et $\mathrm{P} 015$ présentent une réduction de leur

motivation intrinsèque à pratiquer des activités physiques. À l'inverse, les deux patients du groupe GC présentent des changements cliniquement significatifs : le patient P002 présente moins d'amotivation et les patients $\mathrm{P} 002$ et P003 présentent une réduction de leur dépense énergétique (voir tableau 3).

Après six mois de participation, les patients des groupes GI et GIC (P004 et P006 respectivement) n'ont montré de changement significatif ni de leurs profils motivationnels, ni de leurs niveaux d'activité physique, ni de leur sédentarité. Néanmoins, la patiente du groupe GC (P003) présentait une augmentation de sa motivation extrinsèque ainsi qu'une augmentation du nombre de pas effectués dans une journée (voir tableau 3). Ces résultats suggèrent que, malgré des profils motivationnels parfois plus favorables à l'initiation de comportements actifs, des changements de niveaux d'activité physique ne sont pas toujours observés au cours d'une intervention basée sur la pleine conscience.

\section{Pleine conscience et détresse psychologique.}

À l'inclusion, malgré les effectifs inégaux entre les trois groupes de patients $\left(\mathrm{n}_{\mathrm{GI}}=3\right.$; $\mathrm{n}_{\mathrm{GIC}}=6 ; \mathrm{n}_{\mathrm{GC}}=3$ ), certaines tendances sont observées sur les capacités de pleine conscience et la détresse psychologique. En effet, les patients du groupe GI semblent présenter des scores plus élevés de pleine conscience (évalués de manière dispositionnelle avec la MAAS ou situationnelle avec la DMRS) et moins élevés de détresse psychologique en comparaison aux patients des groupes GIC et GC (voir tableau 2).

À un mois de participation, la patiente du groupe GI (P004) n'a montré de changement significatif ni pour les capacités de pleine conscience, ni pour la détresse psychologique. 


\section{PLEINE CONSCIENCE ET BINGE EATING}

571 D'autre part, un patient sur six du groupe GIC (P006) a montré une augmentation

572 cliniquement significative de la détresse psychologique après un mois de participation. Enfin,

573 un patient sur deux du groupe GC (P002) a présenté une augmentation cliniquement

574 significative des scores de dépression et de détresse psychologique à un mois (voir tableau 3).

575 Après six mois de participation, aucun patient n'a montré de changement cliniquement

576 significatif ni pour les capacités de pleine conscience ni pour la détresse psychologique.

577 Cependant, la patiente P004 du groupe GI a montré une tendance à l'amélioration de ses

578 capacités dispositionnelles et à la réduction de ses capacités situationnelles de pleine

579 conscience. D'autre part, la patiente P003 (groupe GC) a présenté une tendance à la réduction

580 de ses capacités dispositionnelles et situationnelles de pleine conscience, malgré une stabilité

581 de la détresse psychologique (voir tableau 3). Ces résultats questionnent sur l'adhésion des

582 participants des groupes GI et GIC, qui pourrait expliquer ce manque de significativité

583 clinique.

584 Adhésion à l'intervention.

585 Au cours du premier mois de participation, correspondant à la période d'écoute

586 structurée des séances, les patients du groupe GI ont écouté $10 \%( \pm 4,71)$ et les patients du

587 groupe GIC ont écouté 30,67\% ( $\pm 18,77)$ des séances prescrites en moyenne. Pendant la

588 période d'écoute autonome, les patients du groupe GI ont écouté 3,83\% $( \pm 5,42)$ et les patients

589 du groupe GIC ont écouté 12,18\% ( \pm 19,11) des séances prescrites en moyenne. Les scores

590 d'adhésion des participants à l'écoute des séances audio sont présentés dans le tableau 3. Les

591 résultats individuels des patients sont présentés en matériel supplémentaire (matériel

592 supplémentaire 2), et mis en relation avec leurs niveaux d'adhésion à l'intervention, sous

593 forme de série de cas cliniques. 


\section{PLEINE CONSCIENCE ET BINGE EATING}

Le but de l'étude MindOb était d'évaluer l'efficacité d'une intervention quotidienne à

597

598

599

600

601

602

603

604

605

606

607

608

609

610

611

612

613

614

615

616

617

618

619

620

distance basée sur la pleine conscience auprès de patients obèses suivis dans un service de nutrition hospitalier et atteints de trouble de binge eating. L'hypothèse principale était que la pleine conscience permettrait de réduire l'impulsivité alimentaire de ces patients. Toutefois, étant donné que l'étude est encore en cours de recrutement, les résultats présentés dans cet article concernent les 16 premiers patients inclus. Ainsi, les résultats ont été présentés sous forme de série de cas contrôlés. Le résultat principal de cette série de cas réside dans le nombre de retraits de consentement et de perdus de vue. En effet, quatre patients ont retiré leur consentement en cours de participation, et trois patients ont été perdus de vue. Ces sept patients représentent $43 \%$ de l'échantillon actuel, ce qui est conséquent compte tenu des données de la littérature [42]. D'autre part, étant donné que seule une patiente tirée au sort dans le groupe expérimental (c-à-d. GI) ne s'est pas retirée de l'étude, l'efficacité de la pleine conscience ne peut pas être appréciée dans cet échantillon. Enfin, les données recueillies à un mois de participation suggèrent qu'une intervention basée sur de la méditation factice [27] pourrait permettre de réduire l'impulsivité alimentaire et l'alimentation émotionnelle de patients souffrant de trouble de binge eating.

Après un mois de participation, dans leur majorité, les patients du groupe contrôle actif (GIC) ont présenté une réduction de leur impulsivité alimentaire et de leur alimentation émotionnelle, tandis que les patients du groupe contrôle inactif (GC) n'ont pas évolué positivement sur ces critères de jugement. Ce résultat suggère que le simple fait d'écouter de la musique relaxante avec pour consigne de «méditer » puisse améliorer les capacités de gestion des émotions de patients obèses avec trouble de binge eating. Cependant, aucun patient de cette étude n'a amélioré significativement ses capacités de pleine conscience, et seulement une patiente du groupe GIC a écouté plus de 50\% des séances prescrites dans le premier mois de participation. Ainsi, il semblerait que les tendances observées soient en partie 


\section{PLEINE CONSCIENCE ET BINGE EATING}

621 attribuables à d'autres facteurs. En effet, les patients du groupe GIC sont majoritairement issus

622 de catégories socioprofessionnelles hautes, ont démarré leur suivi en nutrition peu de temps

623 avant leur inclusion, et ont déjà bénéficié d'un suivi avec un psychologue. Ces caractéristiques

624 présentent des avantages considérables quant à la capacité de ces patients à bénéficier des

625 effets positifs attendus de l'intervention sur les variables liées aux conduites alimentaires. En

626 effet, il a été montré dans la littérature (a) que les individus avec un niveau d'éducation élevé

627 et des catégories socioprofessionnelles hautes bénéficiaient mieux des interventions basées

628 sur la pleine conscience [43,44], (b) qu'un suivi psychologique améliorait les capacités à gérer

629 les pensées et émotions [45] et (c) que le fait d'être dans une démarche de perte de poids (c-à-

630 d. consultation en nutrition) favorisait la motivation des patients à suivre toute intervention

631 thérapeutique leur étant proposée [46].

632 Les résultats liés aux comportements actifs semblent montrer, pour quelques

633 participants du groupe suivant une intervention de méditation factice, une augmentation de la

634 motivation extrinsèque et une diminution de la motivation intrinsèque. Cette tendance

635 observée peut être mise en lien avec les recherches antérieures suggérant qu'en début

636 d'intervention, les individus présentent majoritairement des motivations extrinsèques,

637 favorisant l'initiation d'un comportement [46,47]. Toutefois, la réduction de la motivation

638 intrinsèque est en opposition avec l'interprétation des résultats de Ruffault et al. [22]

639 suggérant que les capacités de pleine conscience renforceraient le lien entre motivation

640 intrinsèque et niveau d'activité physique. En effet, ces résultats observés en population non

641 clinique ne semblent pas s'appliquer à une population en demande d'une prise en charge de

642 l'obésité depuis moins d'un an. D'autre part, étant donné que les participants du groupe GIC

643 n'ont pas présenté d'augmentation de leurs capacités de pleine conscience après un mois de

644 participation, il semblait peu probable que ce lien observé dans la littérature soit également

645 observé dans notre échantillon. 


\section{PLEINE CONSCIENCE ET BINGE EATING}

Par ailleurs, les résultats après un mois de participation montrent une tendance à

l'augmentation des scores de détresse psychologique dans les deux groupes bénéficiant d'une intervention, accompagnée d'une tendance à l'amélioration des capacités de pleine conscience cliniquement non significative. Certains effets indésirables comme l'augmentation du niveau de détresse psychologique sont fréquents dans les interventions basées sur la pleine conscience, étant donné que le changement de perspective face aux pensées et émotions peut nécessiter un temps d'adaptation avant que les participants ne réussissent à accepter ces cognitions et affects [48]. Toutefois, à l'inclusion, on peut observer que les capacités de pleine conscience des trois patients de GI sont plus élevées que celles des neuf patients des deux autres groupes, et que les scores de détresse psychologique des patients de GI sont plus bas que ceux des patients de GIC et de GC. Cette différence aurait probablement pu être évitée si nous avions ajouté un niveau de stratification lors de la randomisation, en nous basant sur les capacités de pleine conscience et la détresse psychologique des participants à l'inclusion. En effet, stratifier la randomisation sur ces deux critères aurait permis d'avoir moins d'hétérogénéité entre les trois groupes expérimentaux. D'autre part, la patiente du groupe GI et le patient du groupe GIC dont les données à six mois de participation sont disponibles montrent une réduction de leur détresse psychologique par rapport à leurs scores à un mois, ainsi qu'une augmentation de leurs capacités de pleine conscience à six mois par rapport à un mois. Ces résultats préliminaires, bien qu'à interpréter avec précaution, pourraient suggérer que l'intervention testée ainsi que l'intervention contrôle permettent d'augmenter les capacités à faire face à des situations difficiles du quotidien à long terme. Les recherches à venir testant l'efficacité d'interventions basées sur la pleine conscience pourraient inclure une évaluation des mécanismes d'action de la pleine conscience comme par exemple la régulation des émotions ou encore les capacités de coping.

Parmi les 16 patients inclus, aucun n'a suivi entièrement l'intervention ni sur le premier 


\section{PLEINE CONSCIENCE ET BINGE EATING}

671 mois, ni après la première visite de suivi. Ce manque d'adhésion de la part des participants est

672 probablement le facteur principal qui pourrait expliquer le peu de résultats cliniquement

673 significatifs observés. Contre toute attente, il semblerait pourtant que les patients avaient

674 l'intention de suivre ce programme d'entraînement à la pleine conscience au moment de la

675 visite d'inclusion. Il est probable que les recueils alimentaires, le carnet de participation et les

676 deux comptes à créer sur les plateformes de l'étude (c-à-d. 31k et ClinShare) aient représentés

677 de grandes difficultés pour les patients. En effet, par exemple, la patiente P001 s'est désistée

678 en partie parce qu'elle se sentait en difficulté face aux démarches préalables au début de

679 l'intervention. Dans la littérature, il est indiqué qu'un suivi de l'adhésion des participants aux

680 programmes d'interventions basées sur la pleine conscience à distance pourrait permettre

681 d'augmenter le niveau d'adhésion des participants [49,50]. Toutefois, Perkins et al. [50]

682 suggèrent que ce type de suivi ayant pour objectif d'améliorer l'adhésion des participants

683 n'améliore pas l'efficacité de l'intervention pour autant. À notre connaissance, l'étude MindOb

684 est la première à mesurer l'adhésion des participants de manière objective avec les données de

685 connexion des participants sur la plateforme 31k. Cependant, lorsqu'il s'agit d'entraînements à

686 la pleine conscience à distance, les études montrent que l'adhésion au protocole expérimental

687 est généralement faible, avec de forts taux d'abandons [52,53] ; et les études qui observent les

688 meilleurs effets sont en fait les études qui n'analysent que les résultats des participants ayant

689 le mieux adhéré à l'intervention [53]. Fish et al. [52] suggèrent que l'incitation à la pratique

690 informelle (p.ex. : focalisation de l'attention sur la respiration dans des situations du

691 quotidien, body scans rapides) pourrait permettre d'améliorer l'adhésion aux programmes de

692 pleine conscience en ligne. Il est également essentiel de noter que, malgré que la majorité des

693 participants semblait apprécier les séances de pleine conscience et de méditation factice, il ne

694 semble pas y avoir eu d'effet sur l'adhésion des participants. Cette observation peut

695 probablement s'expliquer par le manque d'information des patients sur le fonctionnement de 


\section{PLEINE CONSCIENCE ET BINGE EATING}

696

697

698

699

700

701

702

703

704

705

706

707

708

709

710

711

712

713

714

715

716

717

718

719

720

l'intervention testée.

Dans ces résultats préliminaires, l'adhésion des participants à l'intervention et au protocole expérimental, facteur principal pouvant favoriser les changements attendus sur les variables d'intérêt, représente une limite majeure à l'interprétation des résultats. En effet, certains auteurs suggèrent de mettre en place un programme de psychoéducation avant le début du programme de pleine conscience dans le but d'améliorer l'adhésion des participants [54]. En effet, il semblerait que les patients en demande d'une prise en charge pour perdre du poids dans un service de nutrition aient besoin d'acquérir des connaissances sur leurs troubles ainsi que sur les techniques qui leur sont proposées [46]. Ainsi, nous aurions pu ajouter une séance de groupe pour expliquer aux participants ce qu'est le binge eating et en quoi la pleine conscience pourrait les aider. Cependant, nous avons fait le choix de ne pas le faire puisque ce choix aurait pu biaiser les résultats observés en raison du biais de désirabilité sociale induit (cà-d. les patients, sachant ce qui est attendu, auront tendance à répondre aux questionnaires dans le sens des hypothèses). Par ailleurs, une bande son de trois minutes était disponible tous les jours pour les participants des groupes GI et GIC, afin de leur expliquer brièvement en quoi l'intervention pourrait les aider.

Afin d'améliorer l'adhésion aux interventions à distance, Marks et Cavanagh [49] proposent de mettre en place un rappel hebdomadaire afin que les participants puissent se rendre sur la plateforme d'intervention. Encore une fois, c'était un choix de notre part de ne pas relancer les participants systématiquement, afin de ne pas plus les déranger pendant les douze mois de participation, et ainsi observer la mise en place de l'écoute des séances dans leur quotidien. D'autre part, nous aurions pu mettre en place un système permettant d'identifier les patients qui n'ont pas écouté de séance plusieurs jours consécutifs afin de leur rappeler d'écouter tous les jours une séance et de leur donner des conseils. Cette technique aurait pu permettre un meilleur suivi des participants à distance, cependant techniquement il 


\section{PLEINE CONSCIENCE ET BINGE EATING}

721 était impossible de le mettre en place. À l'avenir, les recherches interventionnelles à distance

722 devraient proposer un suivi personnalisé de l'adhésion des participants. Ce type de suivi

723 nécessite d'avoir une mesure en temps réel de l'adhésion des participants.

724 Dans cette étude, la pleine conscience était considérée comme un accompagnement

725 complémentaire et écologique à une prise en charge classique dans un service de nutrition :

726 complémentaire parce que les patients ont besoin à la fois d'un suivi pluridisciplinaire pour

727 leurs problèmes de poids avec un suivi à but psychothérapeutique pour leur trouble de binge

728 eating, et écologique parce que peu de ressources humaines sont nécessaires pour proposer

729 l'intervention aux patients. Le fait que cette intervention soit testée dans le cadre d'une

730 recherche biomédicale a compliqué la tâche des participants. En effet, (a) devoir répondre à

731 l'investigateur qui vérifie les critères d'inclusion et de non-inclusion par téléphone, (b) se

732 rendre à la visite d'inclusion, se faire tirer au sort, recevoir le matériel de l'étude et des

733 explications sur le déroulement de l'étude, (c) devoir remplir un recueil alimentaire ainsi que

734 porter un podomètre pendant sept jours, et (d) devoir répondre à des questionnaires et se

735 rendre à d'autres visites de suivi, représentent une charge organisationnelle lourde pour les

736 patients. Si nous avions simplement mis en place l'intervention en dehors d'un protocole

737 expérimental, les patients n'auraient qu'à se voir expliquer le fonctionnement de la plateforme

738 d'intervention et à se rendre quotidiennement dessus, ce qui aurait pu favoriser leur adhésion.

739 Il est donc particulièrement difficile de tester l'efficacité d'une intervention à distance

740 sans mettre en place des techniques ayant pour objectif d'améliorer l'adhésion des participants.

741 Le simple intérêt des participants pour les techniques de pleine conscience ne semble pas être

742 suffisant pour les faire adhérer, il semble nécessaire pour eux de comprendre comment est-ce

743 que cette technique pourrait les aider. Un protocole expérimental plus simple et moins long

744 pourrait être une solution afin d'adapter l'étude à plus large échelle. Toutefois, certaines

745 tendances en accord avec les hypothèses émises sont observées dans les résultats de cette 


\section{PLEINE CONSCIENCE ET BINGE EATING}

746 étude. Il est donc nécessaire de continuer les inclusions, après avoir modifié le protocole

747 d'intervention en y ajoutant (a) un flyer de psychoéducation, (b) des rappels quotidiens par

748 SMS pendant les quatre premières semaines, ainsi que (c) du support téléphonique

749 hebdomadaire à la demande des participant, de sorte à augmenter la puissance statistique et 750 permettre des analyses inférentielles.

751

752 Conflit d'intérêt : aucun

753 


\section{PLEINE CONSCIENCE ET BINGE EATING}

\section{Références}

755 1. Matta J, Zins M, Feral-Pierssens AL, Carette C, Ozguler A, Goldberg M, et al. Prévalence du surpoids, de l'obésité et des facteurs de risque cardio-métaboliques dans la cohorte Constances. Bull Epidémiologique Hebd 2016;35-36:640-6.

2. Ng M, Fleming T, Robinson M, Thomson B, Graetz N, Margono C, et al. Global, regional, and national prevalence of overweight and obesity in children and adults during 1980-2013: A systematic analysis for the Global Burden of Disease Study 2013. The Lancet 2014;384(9945):766-81.

3. Haute Autorité de Santé. Surpoids et obésité de l'adulte : prise en charge médicale de premier recours. [Internet]. 2011 [cited 2015 Oct 27]. Available from: http://www.hassante.fr

4. World Health Organization. Global status report on noncommunicable diseases 2014 [Internet]. 2014 [cited 2016 Dec 30]. Available from: http://www.who.int/nmh/publications/ncd-status-report-2014/en/

5. Dulloo AG. Explaining the failures of obesity therapy: Willpower attenuation, target miscalculation or metabolic compensation? Int J Obes 2012;36(11):1418-20.

770 6. Godfrey KM, Gallo LC, Afari N. Mindfulness-based interventions for binge eating: A systematic review and meta-analysis. J Behav Med 2015;38(2):348-62.

772 7. Godsey J. The role of mindfulness based interventions in the treatment of obesity and eating disorders: An integrative review. Complement Ther Med 2013;21(4):430-9.

774 8. Katterman SN, Kleinman BM, Hood MM, Nackers LM, Corsica JA. Mindfulness 775 meditation as an intervention for binge eating, emotional eating, and weight loss: A systematic review. Eat Behav 2014;15(2):197-204. 


\section{PLEINE CONSCIENCE ET BINGE EATING}

777 9. O'Reilly GA, Cook L, Spruijt-Metz D, Black DS. Mindfulness-based interventions for obesity-related eating behaviours: a literature review: Mindfulness interventions for eating behaviours. Obes Rev 2014;15(6):453-61.

780

10. Ruffault A, Czernichow S, Hagger MS, Ferrand M, Erichot N, Carette C, et al. The effects of mindfulness training on weight-loss and health-related behaviours in adults with overweight and obesity: A systematic review and meta-analysis. Obes Res Clin Pract 2017;11(5):90-111.

11. Wanden-Berghe RG, Sanz-Valero J, Wanden-Berghe C. The application of mindfulness to eating disorders treatment: A systematic review. Eat Disord 2010;19(1):34-48.

12. American Psychiatric Association. Diagnostic and statistical manual of mental disorders. 5th ed. Washington, USA: American Psychiatric Association; 2013.

13. Kessler RC, Berglund PA, Chiu WT, Deitz AC, Hudson JI, Shahly V, et al. The prevalence and correlates of binge eating disorder in the World Health Organization World Mental Health Surveys. Biol Psychiatry 2013;73(9):904-14.

14. Hagger MS, Panetta G, Leung CM, Wong GG, Wang JCK, Chan DKC, et al. Chronic Inhibition, Self-Control and Eating Behavior: Test of a 'Resource Depletion' Model. PLoS ONE 2013;8(10):e76888.

15. Leehr EJ, Krohmer K, Schag K, Dresler T, Zipfel S, Giel KE. Emotion regulation model in binge eating disorder and obesity: A systematic review. Neurosci Biobehav Rev 2015;49:125-34.

16. Brown KW, Ryan RM. The benefits of being present: Mindfulness and its role in psychological well-being. J Pers Soc Psychol 2003;84(4):822-48.

17. Baer RA. Mindfulness training as a clinical intervention: A conceptual and empirical review. Clin Psychol Sci Pract 2003;10(2):125-43. 


\section{PLEINE CONSCIENCE ET BINGE EATING}

801

802

803

804

805

806

807

808

809

810

18. Kabat-Zinn J. Full catastrophe living: Using the wisdom of your body and mind to face stress, pain and illness. New-York, USA: Dell Publishing; 1990.

19. Baer RA, Fischer S, Huss DB. Mindfulness and acceptance in the treatment of disordered eating. J Ration-Emotive Cogn-Behav Ther 2005;23(4):281-300.

20. Lattimore P, Mead BR, Irwin L, Grice L, Carson R, Malinowski P. 'I can't accept that feeling': Relationships between interoceptive awareness, mindfulness and eating disorder symptoms in females with, and at-risk of an eating disorder. Psychiatry Res 2017;247:163-71.

21. Hendrickson K, Rasmussen EB. Effects of mindful eating training on delay and probability discounting for food and money in obese and healthy-weight individuals. Behav Res Ther 2013;51(7):399-409.

22. Lacaille J, Ly J, Zacchia N, Bourkas S, Glaser E, Knäuper B. The effects of three mindfulness skills on chocolate cravings. Appetite 2014;76:101-12.

23. Ruffault A, Bernier M, Juge N, Fournier JF. Mindfulness may moderate the relationship between intrinsic motivation and physical activity: A cross-sectional study. Mindfulness 2016;7(2):445-52.

24. Chatzisarantis NLD, Hagger MS. Mindfulness and the intention-behavior relationship within the theory of planned behavior. Pers Soc Psychol Bull 2007;33(5):663-76.

25. Butryn ML, Forman E, Hoffman K, Shaw J, Juarascio A. A pilot study of acceptance and commitment therapy for promotion of physical activity. J Phys Act Health 2011;8(4):516-22.

26. Ruffault A, Carette C, Lurbe i Puerto K, Juge N, Beauchet A, Benoliel JJ, et al. Randomized controlled trial of a 12-month computerized mindfulness-based intervention for obese patients with binge eating disorder: The MindOb study protocol. Contemp Clin Trials 2016;49:126-33. 


\section{PLEINE CONSCIENCE ET BINGE EATING}

27. Zeidan F, Johnson SK, Gordon NS, Goolkasian P. Effects of brief and sham mindfulness meditation on mood and cardiovascular variables. J Altern Complement Med 2010;16(8):867-73.

28. Teasdale JD, Segal ZV, Williams JMG, Ridgeway VA, Soulsby JM, Lau MA. Prevention of relapse/recurrence in major depression by Mindfulness-Based Cognitive Therapy. J Consult Clin Psychol 2000;68(4):615-23.

29. Hayes SC, Strosahl K, Wilson KG. Acceptance and commitment therapy : An experiential approach to behavior change. New-York, USA: Guilford Press; 2003.

30. European Values Study. European Values Study 2008: Integrated Dataset (EVS 2008). Cologne, Germany: GESIS Data Archive; 2011.

31. Karlsson J, Persson LO, Sjöström L, Sullivan M. Psychometric properties and factor structure of the Three-Factor Eating Questionnaire (TFEQ) in obese men and women: Results from the Swedish Obese Subjects (SOS) study. Int J Obes 2000;24(12):171525.

32. Cappelleri JC, Bushmakin AG, Gerber RA, Leidy NK, Sexton CC, Lowe MR, et al. Psychometric analysis of the Three-Factor Eating Questionnaire-R21: Results from a large diverse sample of obese and non-obese participants. Int J Obes 2009;33(6):61120.

33. Rutishauser IHE. Dietary intake measurements. Public Health Nutr. 2005;8(7a).

34. Markland D, Tobin V. A modification to the Behavioural Regulation in Exercise Questionnaire to include an assessment of amotivation. J Sport Exerc Psychol 2004;26(2):191-6.

35. Craig CL, Marshall AL, Sjostrom M, Bauman AE, Booth ML, Ainsworth BE, et al. International Physical Activity Questionnaire: 12-Country reliability and validity. Med Sci Sports Exerc 2003;35(8):1381-95. 


\section{PLEINE CONSCIENCE ET BINGE EATING}

851 36. Zigmond AS, Snaith RP. The hospital anxiety and depression scale. Acta Psychiatr Scand 1983;67(6):361-70.

853 37. Bjelland I, Dahl AA, Haug TT, Neckelmann D. The validity of the Hospital Anxiety and Depression Scale. J Psychosom Res 2002;52(2):69-77.

38. Lacaille J, Sadikaj G, Nishioka M, Flanders J, Knäuper B. Measuring mindful responding in daily life: Validation of the Daily Mindful Responding Scale (DMRS). Mindfulness 2015;6(6):1422-36.

39. Rogers CR. Client centred thearpy: Its current practice, implications and theory. London, UK: Constable; 2003.

40. Jacobson NS, Truax P. Clinical significance: A statistical approach to defing meaningful change in psychotherapy research. J Consult Clin Psychol 1991;59(1):12-9.

41. Bost RH, Wen FK, Basso MR, Cates GR. Online tools for evaluating patient change: Statistical foundations, clinical applications, research relevance. Rehabil Psychol 2008;53(3):313-20.

42. Buhrman M, Skoglund A, Husell J, Bergström K, Gordh T, Hursti T, et al. Guided internet-delivered acceptance and commitment therapy for chronic pain patients: A randomized controlled trial. Behav Res Ther 2013;51(6):307-15.

43. Kabat-Zinn J, Chapman-Waldrop A. Compliance with an outpatient stress reduction program: Rates and predictors of program completion. J Behav Med 1988;11(4):33352.

871 44. Tovote KA, Schroevers MJ, Snippe E, Emmelkamp PMG, Links TP, Sanderman R, et 872 al. What works best for whom? Cognitive Behavior Therapy and Mindfulness-Based 873 Cognitive Therapy for depressive symptoms in patients with diabetes. PLOS ONE 2017;12(6):e0179941. 


\section{PLEINE CONSCIENCE ET BINGE EATING}

875 45. Kazdin AE. Mediators and mechanisms of change in psychotherapy research. Annu Rev Clin Psychol 2007;3(1):1-27.

877 46. Rogerson D, Soltani H, Copeland R. The weight-loss experience: A qualitative exploration. BMC Public Health 2016;16(1):371.

879 47. Hagger MS, Chatzisarantis NLD. Intrinsic motivation and self-determination in exercise and sport. Champaign, US: Human Kinetics; 2007.

48. Creswell JD. Mindfulness Interventions. Annu Rev Psychol. 2017;68(1):491-516.

49. Kvillemo P, Brandberg Y, Bränström R. Feasibility and outcomes of an internet-based mindfulness training program: A pilot randomized controlled trial. JMIR Ment Health 2016;3(3):e33.

50. Marks I, Cavanagh K. Computer-aided psychological treatments: Evolving issues. Annu Rev Clin Psychol 2009;5(1):121-41.

51. Perkins SSJ, Murphy RRM, Schmidt UUS, Williams C, Cochrane Common Mental Database Syst Rev 2006.

52. Cavanagh K, Strauss C, Forder L, Jones F. Can mindfulness and acceptance be learnt by self-help? A systematic review and meta-analysis of mindfulness and acceptance-based self-help interventions. Clin Psychol Rev 2014;34(2):118-29.

53. Fish J, Brimson J, Lynch S. Mindfulness interventions delivered by technology without facilitator involvement: What research exists and what are the clinical outcomes? Mindfulness 2016;7(5):1011-23.

54. Pinto-Gouveia J, Carvalho SA, Palmeira L, Castilho P, Duarte C, Ferreira C, et al. Incorporating psychoeducation, mindfulness and self-compassion in a new programme for binge eating (BEfree): Exploring processes of change. J Health Psychol 2016. 


\section{PLEINE CONSCIENCE ET BINGE EATING}

\section{$900 \quad$ Titres et légendes des figures}

901 Figure 1. Représentation schématique de la procédure de l'étude.

902 Figure 1. Graphical representation of the study procedure.

903

904 Figure 2. Diagramme de flux de participation.

905 Figure 2. Flow chart of participation.

906 


\section{Condition expérimentale : Pleine conscience}

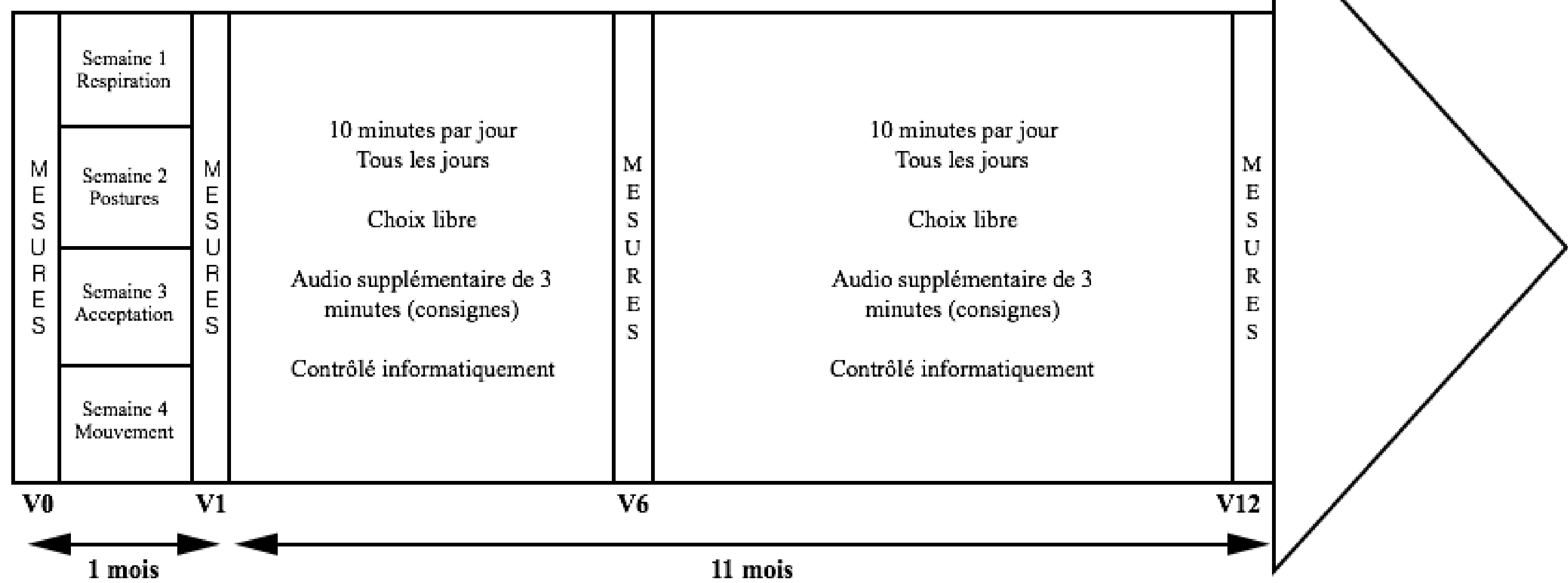

\section{Condition contrôle actif : Méditation factice}

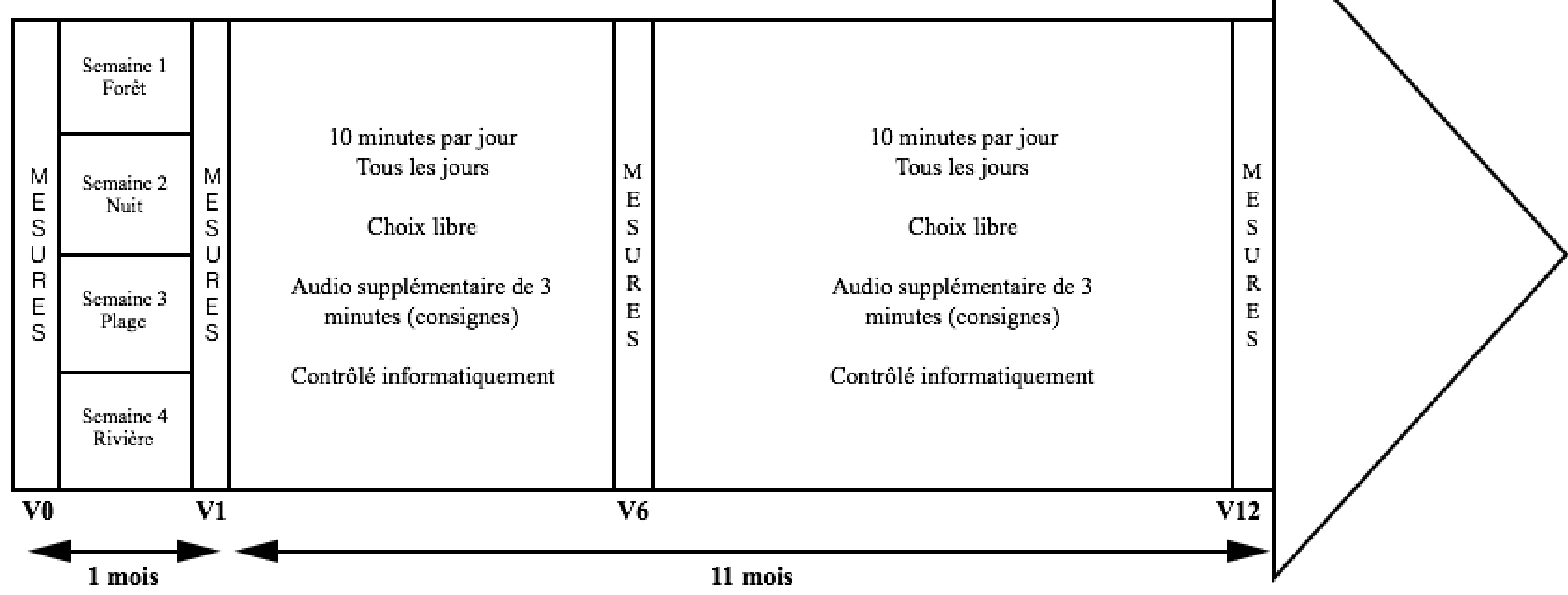


Tableau 1. Caractéristiques des participants.

Table 1. Participants' caracteristics.

\begin{tabular}{|c|c|c|c|c|c|c|c|c|}
\hline $\begin{array}{l}\text { Numéro } \\
\text { (groupe) }\end{array}$ & $\begin{array}{l}\text { Genre, âge, } \\
\text { IMC }\end{array}$ & Comorbidités & $\begin{array}{c}\text { Statut } \\
\text { pondéral }\end{array}$ & $\begin{array}{c}\text { Diplôme, CSP, } \\
\text { emploi }\end{array}$ & Vie conjugale & Santé mentale & $\begin{array}{c}\text { Activités } \\
\text { physiques }\end{array}$ & Autres \\
\hline $\begin{array}{l}\text { P001 (GI, } \\
\text { abandon) }\end{array}$ & $\begin{array}{l}\text { Femme, } 52 \text { ans, } \\
40,48 \mathrm{~kg} / \mathrm{m}^{2}\end{array}$ & $\begin{array}{l}\text { HTA, SAS, } \\
\text { DOA }\end{array}$ & $\begin{array}{l}\text { Actuellement } \\
\text { stable. Début à } \\
\text { l'âge adulte. Pas } \\
\text { de régimes. PEC } \\
\text { depuis plus d'un } \\
\text { an. IMC } \\
\text { maximal atteint } \\
48,44 \text { (2013). } \\
\text { IMC idéal } \\
27.68 \text {. }\end{array}$ & $\begin{array}{l}\text { Niveau Bac+3. } \\
\text { Employée de la } \\
\text { fonction } \\
\text { publique. } \\
\text { Actuellement au } \\
\text { chômage. }\end{array}$ & $\begin{array}{l}\text { Vit avec son } \\
\text { conjoint ( } 50 \\
\text { ans) et } 2 \\
\text { enfants. }\end{array}$ & $\begin{array}{l}\text { Bipolarité, } \\
\text { troubles } \\
\text { anxieux, TS. } \\
\text { Suivie en CMP } \\
\text { depuis } 2010 .\end{array}$ & $\begin{array}{l}\text { Ne fait plus de } \\
\text { sport. Ne se } \\
\text { décrit pas } \\
\text { comme active. } \\
\text { Décision de se } \\
\text { remettre à être } \\
\text { active } \\
\text { rapidement. }\end{array}$ & $\begin{array}{l}\text { Se décrit comme } \\
\text { quelqu'un } \\
\text { d'accessible à la } \\
\text { spiritualité, et } \\
\text { pratique au moins } \\
\text { une fois par mois. } \\
\text { AInf=7/7. } \\
\mathrm{INT}_{\text {diet }}=10 / 14 \text {. } \\
\mathrm{INT}_{\mathrm{AP}}=10 / 14 \text {. } \\
\mathrm{INT}_{\text {fy }}=7 / 7 \text {. }\end{array}$ \\
\hline P002 (GC) & $\begin{array}{l}\text { Homme, } 20 \text { ans, } \\
44,59 \mathrm{~kg} / \mathrm{m}^{2}\end{array}$ & Aucune & $\begin{array}{l}\text { Actuellement } \\
\text { stable. Début } \\
\text { dans l'enfance. } \\
\text { Nombreux } \\
\text { régimes. PEC } \\
\text { depuis plus d'un } \\
\text { an. IMC } \\
\text { maximal atteint } \\
47,23 \text { (2015). } \\
\text { IMC idéal } \\
32,70 \text {. }\end{array}$ & $\begin{array}{l}\text { Niveau Bac+3. } \\
\text { Artisan } \\
\text { commerçant en } \\
\text { CDD à temps } \\
\text { plein. }\end{array}$ & $\begin{array}{l}\text { Célibataire sans } \\
\text { enfants à } \\
\text { charge. Vit chez } \\
\text { ses parents. }\end{array}$ & $\begin{array}{l}\text { Pas } \\
\text { d'antécédents } \\
\text { psychiatriques. } \\
\text { Consulte un } \\
\text { psychologue } \\
\text { régulièrement } \\
\text { depuis } 2 \text { ans. }\end{array}$ & $\begin{array}{l}\text { Pratique un } \\
\text { sport et se décrit } \\
\text { comme actif } \\
\text { depuis } 10 \text { ans. }\end{array}$ & $\begin{array}{l}\text { Se décrit comme } \\
\text { quelqu'un } \\
\text { d'accessible à la } \\
\text { spiritualité. } \\
\text { AInf }=7 / 7 \\
\text { INT }_{\text {diet }}=8 / 14 \\
\text { INT }_{\text {AP }}=11 / 14 \\
\text { INT }_{\text {psy }}=6 / 7\end{array}$ \\
\hline P003 (GC) & $\begin{array}{l}\text { Femme, } 44 \text { ans, } \\
39,06 \mathrm{~kg} / \mathrm{m}^{2}\end{array}$ & DT2, cancer & $\begin{array}{l}\text { Actuellement } \\
\text { descendant. } \\
\text { Début à l'âge } \\
\text { adulte. } \\
\text { Quelques } \\
\text { régimes. PEC } \\
\text { depuis plus d'un } \\
\text { an. IMC } \\
\text { maximal atteint } \\
\text { 41,80 (2016). } \\
\text { IMC idéal } \\
\text { 27,34. }\end{array}$ & $\begin{array}{l}\text { Niveau Bac+3. } \\
\text { Employée } \\
\text { administratif } \\
\text { d'entreprise en } \\
\text { CDI à temps } \\
\text { partiel. }\end{array}$ & $\begin{array}{l}\text { Célibataire avec } \\
2 \text { enfants à } \\
\text { charge. Vit } \\
\text { seule. }\end{array}$ & $\begin{array}{l}\text { Pas } \\
\text { d'antécédents } \\
\text { psychiatriques. } \\
\text { N'a jamais } \\
\text { consulté de } \\
\text { psychologue ni } \\
\text { de psychiatre. }\end{array}$ & $\begin{array}{l}\text { Ne fait plus de } \\
\text { sport. Se décrit } \\
\text { comme active } \\
\text { depuis son } \\
\text { adolescence. }\end{array}$ & $\begin{array}{l}\text { Ne se décrit pas } \\
\text { comme quelqu'un } \\
\text { d'accessible à la } \\
\text { spiritualité. } \\
\text { AInf }=7 / 7 \\
\mathrm{INT}_{\text {diet }}=12 / 14 \text {. } \\
\mathrm{INT}_{\mathrm{AP}}=12 / 14 \text {. } \\
\mathrm{INT}_{\mathrm{psy}}=4 / 7\end{array}$ \\
\hline
\end{tabular}




\begin{tabular}{|c|c|c|c|c|c|c|c|c|}
\hline P004 (GI) & $\begin{array}{l}\text { Femme, } 24 \text { ans, } \\
35,25 \mathrm{~kg} / \mathrm{m}^{2}\end{array}$ & Asthme & $\begin{array}{l}\text { Actuellement } \\
\text { stable. Début } \\
\text { dans l'enfance. } \\
\text { Pas de régimes. } \\
\text { PEC depuis plus } \\
\text { d'un an. IMC } \\
\text { maximal atteint } \\
\text { 37,65 (2014). } \\
\text { IMC idéal } \\
\text { 28,04. }\end{array}$ & $\begin{array}{l}\text { Niveau brevet } \\
\text { des collèges. } \\
\text { Employée de la } \\
\text { fonction } \\
\text { publique au } \\
\text { chômage depuis } \\
4 \text { ans. }\end{array}$ & $\begin{array}{l}\text { Célibataire sans } \\
\text { enfant à charge. } \\
\text { Vit chez ses } \\
\text { parents. }\end{array}$ & $\begin{array}{l}\text { Épisodes } \\
\text { dépressifs, } \\
\text { troubles } \\
\text { anxieux, TS. } \\
\text { Suivie par un } \\
\text { pédopsychiatre } \\
\text { depuis } 10 \text { ans. }\end{array}$ & $\begin{array}{l}\text { Fait du sport et } \\
\text { se décrit comme } \\
\text { active depuis } 8 \\
\text { ans. }\end{array}$ & $\begin{array}{l}\text { Ne se décrit pas } \\
\text { comme quelqu'un } \\
\text { d'accessible à la } \\
\text { spiritualité. } \\
\text { AInf=3/7. } \\
\mathrm{INT}_{\text {diet }}=8 / 14 \text {. } \\
\mathrm{INT}_{\mathrm{AP}}=10 / 14 \text {. } \\
\mathrm{INT}_{\mathrm{psy}}=6 / 7 .\end{array}$ \\
\hline $\begin{array}{l}\text { P005 (GI, } \\
\text { perdu de vue) }\end{array}$ & $\begin{array}{l}\text { Homme, } 24 \text { ans, } \\
43,03 \mathrm{~kg} / \mathrm{m}^{2}\end{array}$ & HTA, DOA & $\begin{array}{l}\text { Actuellement } \\
\text { descendant. } \\
\text { Début dans } \\
\text { l'enfance. } \\
\text { Quelques } \\
\text { régimes. PEC } \\
\text { depuis moins de } \\
3 \text { mois. IMC } \\
\text { maximal atteint } \\
53,78 \text { (2010). } \\
\text { IMC idéal } \\
\text { 34,06. }\end{array}$ & $\begin{array}{l}\text { Sans diplôme. } \\
\text { Employé de la } \\
\text { fonction } \\
\text { publique en CDI } \\
\text { à temps plein. }\end{array}$ & $\begin{array}{l}\text { Vit avec sa } \\
\text { conjointe ( } 23 \\
\text { ans) sans enfant. }\end{array}$ & $\begin{array}{l}\text { Dépression, } \\
\text { trouble anxieux. } \\
\text { N'a jamais vu } \\
\text { de psychologue } \\
\text { pour un suivi. }\end{array}$ & $\begin{array}{l}\text { Fait du sport et } \\
\text { se décrit comme } \\
\text { actif depuis } 2 \\
\text { ans. }\end{array}$ & $\begin{array}{l}\text { Ne se décrit pas } \\
\text { comme quelqu'un } \\
\text { d'accessible à la } \\
\text { spiritualité. } \\
\text { AInf=5/7. } \\
\mathrm{INT}_{\text {diet }}=6 / 14 \text {. } \\
\mathrm{INT}_{\mathrm{AP}}=8 / 14 . \\
\mathrm{INT}_{\mathrm{psy}}=6 / 7 .\end{array}$ \\
\hline P006 (GIC) & $\begin{array}{l}\text { Homme, } 55 \text { ans, } \\
41,58 \mathrm{~kg} / \mathrm{m}^{2}\end{array}$ & $\begin{array}{l}\text { HTA, SAS, } \\
\text { asthme }\end{array}$ & $\begin{array}{l}\text { Actuellement } \\
\text { stable. Début à } \\
\text { l'âge adulte. } \\
\text { Nombreux } \\
\text { régimes. PEC } \\
\text { depuis moins } \\
\text { d'un an. IMC } \\
\text { maximal atteint } \\
42,93 \text { (2016). } \\
\text { IMC idéal } \\
\text { 33,80. }\end{array}$ & $\begin{array}{l}\text { Niveau Bac+3. } \\
\text { Profession } \\
\text { intermédiaire } \\
\text { des entreprises } \\
\text { en CDI à temps } \\
\text { plein. }\end{array}$ & $\begin{array}{l}\text { Vit en couple } \\
\text { avec son } \\
\text { conjoint ( } 40 \\
\text { ans) sans enfant. }\end{array}$ & $\begin{array}{l}\text { Bipolarité, } \\
\text { trouble anxieux. } \\
\text { A été suivi en } \\
\text { CMP. } \\
\text { S'intéresse à la } \\
\text { pleine } \\
\text { conscience pour } \\
\text { son bien-être. }\end{array}$ & $\begin{array}{l}\text { Ne fait pas de } \\
\text { sport et ne se } \\
\text { décrit pas } \\
\text { comme } \\
\text { quelqu'un } \\
\text { d'actif. } \\
\text { Réfléchit à la } \\
\text { possibilité de } \\
\text { faire de } \\
\text { l'activité } \\
\text { physique, mais } \\
\text { n'est pas encore } \\
\text { décidé. }\end{array}$ & $\begin{array}{l}\text { Se décrit comme } \\
\text { quelqu'un } \\
\text { d'accessible à la } \\
\text { spiritualité et } \\
\text { pratique chaque } \\
\text { semaine. } \\
\text { AInf=7/7. } \\
\mathrm{INT}_{\text {diet }}=10 / 14 \text {. } \\
\mathrm{INT}_{\mathrm{AP}}=4 / 14 \text {. } \\
\mathrm{INT}_{\mathrm{psy}}=6 / 7\end{array}$ \\
\hline
\end{tabular}




\begin{tabular}{|c|c|c|c|c|c|c|c|c|}
\hline $\begin{array}{l}\text { P007 (GIC, } \\
\text { abandon) }\end{array}$ & $\begin{array}{l}\text { Femme, } 58 \text { ans, } \\
33,31 \mathrm{~kg} / \mathrm{m}^{2}\end{array}$ & DOA & $\begin{array}{l}\text { Actuellement } \\
\text { stable. Début } \\
\text { dans l'enfance. } \\
\text { Nombreux } \\
\text { régimes. PEC } \\
\text { depuis plus d'un } \\
\text { an. IMC } \\
\text { maximal atteint } \\
36,68 \text { (2016). } \\
\text { IMC idéal } \\
25,30 \text {. }\end{array}$ & $\begin{array}{l}\text { Diplôme } \\
\text { professionnel. } \\
\text { Employée de } \\
\text { commerce en } \\
\text { CDI à temps } \\
\text { partiel. }\end{array}$ & $\begin{array}{l}\text { Vit seule, } \\
\text { séparée de son } \\
\text { conjoint sans } \\
\text { enfant à charge. }\end{array}$ & $\begin{array}{l}\text { Pas } \\
\text { d'antécédents } \\
\text { psychiatriques. } \\
\text { N'a jamais } \\
\text { consulté de } \\
\text { psychologue ni } \\
\text { de psychiatre. }\end{array}$ & $\begin{array}{l}\text { Fait du sport et } \\
\text { se décrit comme } \\
\text { active depuis } 1 \\
\text { an. }\end{array}$ & $\begin{array}{l}\text { Se décrit comme } \\
\text { quelqu'un } \\
\text { d'accessible à la } \\
\text { spiritualité et } \\
\text { pratique une fois } \\
\text { par semaine. } \\
\text { AInf=7/7. } \\
\mathrm{INT}_{\text {diet }}=11 / 14 \text {. } \\
\mathrm{INT}_{\mathrm{AP}}=11 / 14 \\
\mathrm{INT}_{\mathrm{psy}}=7 / 7\end{array}$ \\
\hline $\begin{array}{l}\text { P008 (GIC, } \\
\text { abandon) }\end{array}$ & $\begin{array}{l}\text { Femme, } 34 \text { ans, } \\
34,11 \mathrm{~kg} / \mathrm{m}^{2}\end{array}$ & Aucune & $\begin{array}{l}\text { Actuellement } \\
\text { stable. Début } \\
\text { dans l'enfance. } \\
\text { Pas de régimes. } \\
\text { PEC depuis } \\
\text { moins d'un } \\
\text { mois. IMC } \\
\text { maximal atteint } \\
34,11 \text { (2016). } \\
\text { IMC idéal } \\
25,40 \text {. }\end{array}$ & $\begin{array}{l}\text { Niveau Bac+3. } \\
\text { Employée } \\
\text { administratif } \\
\text { d'entreprise en } \\
\text { CDI à temps } \\
\text { plein. }\end{array}$ & $\begin{array}{l}\text { Vit avec son } \\
\text { conjoint ( } 37 \\
\text { ans), } 2 \text { enfants à } \\
\text { charge. }\end{array}$ & $\begin{array}{l}\text { Trouble } \\
\text { dépressif. Suivie } \\
\text { depuis } 2,5 \text { ans } \\
\text { par un } \\
\text { psychologue. }\end{array}$ & $\begin{array}{l}\text { Ne fait pas de } \\
\text { sport. Se décrit } \\
\text { comme active } \\
\text { depuis } \\
\text { l'enfance. }\end{array}$ & $\begin{array}{l}\text { Ne se décrit pas } \\
\text { comme quelqu'un } \\
\text { d'accessible à la } \\
\text { spiritualité. } \\
\text { AInf=6/7. } \\
\mathrm{INT}_{\text {diet }}=10 / 14 \text {. } \\
\mathrm{INT}_{\mathrm{AP}}=8 / 14 \text {. } \\
\mathrm{INT}_{\mathrm{psy}}=5 / 7\end{array}$ \\
\hline P009 (GC) & $\begin{array}{l}\text { Homme, } 53 \text { ans, } \\
39,11 \mathrm{~kg} / \mathrm{m}^{2}\end{array}$ & $\begin{array}{l}\text { DT2, MCV, } \\
\text { DOA, asthme }\end{array}$ & $\begin{array}{l}\text { Actuellement } \\
\text { stable. Début à } \\
\text { l'âge adulte. } \\
\text { Quelques } \\
\text { régimes. PEC } \\
\text { depuis moins de } \\
3 \text { mois. IMC } \\
\text { maximal atteint } \\
39,71 \text { (2015). } \\
\text { IMC idéal } \\
29,86 \text {. }\end{array}$ & $\begin{array}{l}\text { Niveau Bac+3. } \\
\text { Chef } \\
\text { d'entreprise en } \\
\text { transition. }\end{array}$ & $\begin{array}{l}\text { Vit avec sa } \\
\text { conjointe }(40 \\
\text { ans) avec } 5 \\
\text { enfants à } \\
\text { charge. }\end{array}$ & $\begin{array}{l}\text { Trouble } \\
\text { dépressif. A } \\
\text { déjà bénéficié } \\
\text { d'un suivi } \\
\text { psychologique. }\end{array}$ & $\begin{array}{l}\text { Fait du sport } \\
\text { mais ne se décrit } \\
\text { pas comme } \\
\text { actif. A décidé } \\
\text { de recommencer } \\
\text { à faire } \\
\text { régulièrement } \\
\text { de l'activité } \\
\text { physique. }\end{array}$ & $\begin{array}{l}\text { Se décrit comme } \\
\text { quelqu'un } \\
\text { d'accessible à la } \\
\text { spiritualité et } \\
\text { pratique chaque } \\
\text { semaine. } \\
\text { AInf=4/7. } \\
\mathrm{INT}_{\text {diet }}=10 / 14 \text {. } \\
\mathrm{INT}_{\mathrm{AP}}=12 / 14 \text {. } \\
\mathrm{INT}_{\mathrm{psy}}=7 / 7\end{array}$ \\
\hline
\end{tabular}




\begin{tabular}{|c|c|c|c|c|c|c|c|c|}
\hline $\begin{array}{l}\text { P010 (GI, } \\
\text { abandon) }\end{array}$ & $\begin{array}{l}\text { Femme, } 32 \text { ans, } \\
31,64 \mathrm{~kg} / \mathrm{m}^{2}\end{array}$ & $\begin{array}{l}\text { SAS, HTA, } \\
\text { DOA, asthme }\end{array}$ & $\begin{array}{l}\text { Actuellement } \\
\text { descendant. } \\
\text { Début dans } \\
\text { l'enfance. } \\
\text { Nombreux } \\
\text { régimes. PEC } \\
\text { depuis moins de } \\
3 \text { mois. IMC } \\
\text { maximal atteint } \\
\text { 35,16 (2016). } \\
\text { IMC idéal } \\
\text { 23,44. }\end{array}$ & $\begin{array}{l}\text { Niveau Bac+3. } \\
\text { Artisan à son } \\
\text { compte. }\end{array}$ & $\begin{array}{l}\text { Vit avec son } \\
\text { conjoint ( } 37 \\
\text { ans) sans enfant } \\
\text { à charge. }\end{array}$ & $\begin{array}{l}\text { Pas } \\
\text { d'antécédents } \\
\text { psychiatriques. } \\
\text { N'a jamais } \\
\text { consulté de } \\
\text { psychologue ni } \\
\text { de psychiatre. }\end{array}$ & $\begin{array}{l}\text { Fait du sport } \\
\text { mais ne se décrit } \\
\text { pas comme } \\
\text { active. Avait } \\
\text { arrêté, mais est } \\
\text { décidée de } \\
\text { rapidement se } \\
\text { remettre à faire } \\
\text { régulièrement } \\
\text { de l'activité } \\
\text { physique. }\end{array}$ & $\begin{array}{l}\text { Se décrit comme } \\
\text { quelqu'un } \\
\text { d'accessible à la } \\
\text { spiritualité et } \\
\text { pratique chaque } \\
\text { semaine. } \\
\text { AInf=7/7. } \\
\mathrm{INT}_{\text {diet }}=13 / 14 \text {. } \\
\mathrm{INT}_{\mathrm{AP}}=10 / 14 \text {. } \\
\mathrm{INT}_{\mathrm{psy}}=5 / 7\end{array}$ \\
\hline $\begin{array}{l}\text { P011 (GIC, } \\
\text { perdue de vue) }\end{array}$ & $\begin{array}{l}\text { Femme, } 34 \text { ans, } \\
32,42 \mathrm{~kg} / \mathrm{m}^{2}\end{array}$ & Aucune & $\begin{array}{l}\text { Actuellement } \\
\text { stable. Début à } \\
\text { l'âge adulte. Pas } \\
\text { de régime. PEC } \\
\text { depuis moins } \\
\text { d'un mois. IMC } \\
\text { maximal atteint } \\
\text { 37,50 (2016). } \\
\text { IMC idéal } \\
\text { 27,34. }\end{array}$ & $\begin{array}{l}\text { Niveau brevet } \\
\text { des collèges. } \\
\text { Employée de } \\
\text { commerce } \\
\text { actuellement au } \\
\text { chômage. }\end{array}$ & $\begin{array}{l}\text { Vit avec son } \\
\text { conjoint ( } 45 \\
\text { ans) avec } 3 \\
\text { enfants à } \\
\text { charge. }\end{array}$ & $\begin{array}{l}\text { Pas } \\
\text { d'antécédents } \\
\text { psychiatriques. } \\
\text { N'a jamais } \\
\text { consulté de } \\
\text { psychologue ni } \\
\text { de psychiatre. }\end{array}$ & $\begin{array}{l}\text { Fait du sport et } \\
\text { se décrit comme } \\
\text { active depuis } \\
\text { l'enfance. }\end{array}$ & $\begin{array}{l}\text { Se décrit comme } \\
\text { quelqu'un } \\
\text { d'accessible à la } \\
\text { spiritualité et } \\
\text { pratique plusieurs } \\
\text { fois par jour. } \\
\text { AInf }=5 / 7 \text {. } \\
\mathrm{INT}_{\text {diet }}=5 / 14 \text {. } \\
\mathrm{INT}_{\mathrm{AP}}=8 / 14 \text {. } \\
\mathrm{INT}_{\mathrm{psy}}=7 / 7 \text {. }\end{array}$ \\
\hline P012 (GI) & $\begin{array}{l}\text { Femme, } 22 \text { ans, } \\
34,19 \mathrm{~kg} / \mathrm{m}^{2}\end{array}$ & DT2 & $\begin{array}{l}\text { Actuellement } \\
\text { descendant. } \\
\text { Début dans } \\
\text { l'enfance. Pas } \\
\text { de régime. PEC } \\
\text { depuis moins } \\
\text { d'un mois. IMC } \\
\text { maximal atteint } \\
\text { 36,63 (2016). } \\
\text { IMC idéal } \\
24,42 \text {. }\end{array}$ & $\begin{array}{l}\text { Diplôme } \\
\text { professionnel. } \\
\text { Employée de la } \\
\text { fonction } \\
\text { publique en } \\
\text { CDD à temps } \\
\text { plein. }\end{array}$ & $\begin{array}{l}\text { Célibataire sans } \\
\text { enfant à charge. } \\
\text { Vit chez ses } \\
\text { parents. }\end{array}$ & $\begin{array}{l}\text { Trouble } \\
\text { dépressif. Suivie } \\
\text { par un } \\
\text { psychologue } \\
\text { depuis } 5 \text { ans. }\end{array}$ & $\begin{array}{l}\text { Fait du sport et } \\
\text { se décrit comme } \\
\text { active depuis } 2 \\
\text { ans. }\end{array}$ & $\begin{array}{l}\text { Ne pense pas être } \\
\text { quelqu'un } \\
\text { d'accessible à la } \\
\text { spiritualité. } \\
\text { AInf=5/7. } \\
\mathrm{INT}_{\text {diet }}=8 / 14 \text {. } \\
\mathrm{INT}_{\mathrm{AP}}=12 / 14 \text {. } \\
\mathrm{INT}_{\mathrm{psy}}=7 / 7 .\end{array}$ \\
\hline
\end{tabular}




\begin{tabular}{|c|c|c|c|c|c|c|c|c|}
\hline P013 (GIC) & $\begin{array}{l}\text { Femme, } 42 \text { ans, } \\
40,63 \mathrm{~kg} / \mathrm{m}^{2}\end{array}$ & Aucune & $\begin{array}{l}\text { Actuellement } \\
\text { stable. Début } \\
\text { dans l'enfance. } \\
\text { Quelques } \\
\text { régimes. PEC } \\
\text { depuis moins de } \\
3 \text { mois. IMC } \\
\text { maximal atteint } \\
42,28 \text { (2016). } \\
\text { IMC idéal } \\
28,08 \text {. }\end{array}$ & $\begin{array}{l}\text { Niveau Bac+5. } \\
\text { Cadre } \\
\text { d'entreprise en } \\
\text { CDI à temps } \\
\text { partiel. }\end{array}$ & $\begin{array}{l}\text { Vit avec son } \\
\text { conjoint ( } 42 \\
\text { ans) avec } 2 \\
\text { enfants à } \\
\text { charge. }\end{array}$ & $\begin{array}{l}\text { Trouble } \\
\text { dépressif. Suivie } \\
\text { depuis } 3 \text { ans par } \\
\text { un psychologue. }\end{array}$ & $\begin{array}{l}\text { Ne fait plus de } \\
\text { sport. Se décrit } \\
\text { comme active. }\end{array}$ & $\begin{array}{l}\text { Ne se décrit pas } \\
\text { comme quelqu'un } \\
\text { d'accessible à la } \\
\text { spiritualité. } \\
\text { AInf }=5 / 7 \\
\text { INT }_{\text {diet }}=11 / 14 \text {. } \\
\text { INT }_{\mathrm{AP}}=12 / 14 \\
\mathrm{INT}_{\mathrm{psy}}=7 / 7\end{array}$ \\
\hline $\begin{array}{l}\text { P014 (GIC, } \\
\text { perdu de vue) }\end{array}$ & $\begin{array}{l}\text { Homme, } 53 \text { ans, } \\
33,15 \mathrm{~kg} / \mathrm{m}^{2}\end{array}$ & $\begin{array}{l}\text { MCV, SAS, } \\
\text { DOA }\end{array}$ & $\begin{array}{l}\text { Actuellement } \\
\text { descendant. } \\
\text { Début à l'âge } \\
\text { adulte. } \\
\text { Quelques } \\
\text { régimes. PEC } \\
\text { depuis moins de } \\
3 \text { mois. IMC } \\
\text { maximal atteint } \\
35,83 \text { (2016). } \\
\text { IMC idéal } \\
25,38 \text {. }\end{array}$ & $\begin{array}{l}\text { Niveau Bac+5. } \\
\text { Cadre } \\
\text { d'entreprise en } \\
\text { CDI à temps } \\
\text { plein. }\end{array}$ & $\begin{array}{l}\text { Vit avec sa } \\
\text { conjointe ( } 57 \\
\text { ans) sans enfant } \\
\text { à charge. }\end{array}$ & $\begin{array}{l}\text { Troubles } \\
\text { anxieux, } \\
\text { addiction, TS. A } \\
\text { été suivi par un } \\
\text { psychologue. } 2 \\
\text { hospitalisations } \\
\text { en psychiatrie } \\
\text { (TS, addiction). }\end{array}$ & $\begin{array}{l}\text { Ne fait plus de } \\
\text { sport et se décrit } \\
\text { comme actif } \\
\text { depuis toujours. }\end{array}$ & $\begin{array}{l}\text { Se décrit comme } \\
\text { quelqu'un } \\
\text { d'accessible à la } \\
\text { spiritualité et } \\
\text { pratique } \\
\text { occasionnellement. } \\
\text { AInf=7/7. } \\
\mathrm{INT}_{\text {diet }}=14 / 14 \text {. } \\
\mathrm{INT}_{\mathrm{AP}}=12 / 14 \text {. } \\
\mathrm{INT}_{\mathrm{psy}}=7 / 7 .\end{array}$ \\
\hline P015 (GIC) & $\begin{array}{l}\text { Homme, } 64 \text { ans, } \\
34,34 \mathrm{~kg} / \mathrm{m}^{2}\end{array}$ & $\begin{array}{l}\text { DT2, MCV, } \\
\text { SAS, DOA, } \\
\text { VIH }\end{array}$ & $\begin{array}{l}\text { Actuellement } \\
\text { descendant. } \\
\text { Début à l'âge } \\
\text { adulte. } \\
\text { Quelques } \\
\text { régimes. PEC } \\
\text { depuis moins de } \\
3 \text { mois. IMC } \\
\text { maximal atteint } \\
35,83 \text { (2016). } \\
\text { IMC idéal } \\
26,87 \text {. }\end{array}$ & $\begin{array}{l}\text { Niveau Bac+3. } \\
\text { Cadre de la } \\
\text { fonction } \\
\text { publique à la } \\
\text { retraite. }\end{array}$ & $\begin{array}{l}\text { Vit avec sa } \\
\text { conjointe (64 } \\
\text { ans) avec un } \\
\text { enfant à charge. }\end{array}$ & $\begin{array}{l}\text { Bipolarité, } \\
\text { trouble anxieux. } \\
\text { A bénéficié } \\
\text { d'un suivi avec } \\
\text { un psychiatre. }\end{array}$ & $\begin{array}{l}\text { Ne fait pas de } \\
\text { sport. Se décrit } \\
\text { comme actif } \\
\text { depuis toujours. }\end{array}$ & $\begin{array}{l}\text { Se décrit comme } \\
\text { quelqu'un } \\
\text { d'accessible à la } \\
\text { spiritualité et } \\
\text { pratique } \\
\text { occasionnellement. } \\
\text { AInf=7/7. } \\
\mathrm{INT}_{\text {diet }}=14 / 14 \text {. } \\
\mathrm{INT}_{\mathrm{AP}}=14 / 14 . \\
\mathrm{INT}_{\mathrm{psy}}=7 / 7 .\end{array}$ \\
\hline
\end{tabular}




\begin{tabular}{|c|c|c|c|c|c|c|c|c|}
\hline P016 (GIC) & $\begin{array}{l}\text { Femme, } 30 \text { ans, } \\
33,31 \mathrm{~kg} / \mathrm{m}^{2}\end{array}$ & DOA & $\begin{array}{l}\text { Actuellement } \\
\text { stable. Début } \\
\text { dans l'enfance. } \\
\text { Pas de régime. } \\
\text { PEC depuis } \\
\text { moins d'un } \\
\text { mois. IMC } \\
\text { maximal atteint } \\
\text { 37,55 (2009). } \\
\text { IMC idéal } \\
\text { 26,12. }\end{array}$ & $\begin{array}{l}\text { Niveau Bac+5. } \\
\text { Cadre } \\
\text { d'entreprise en } \\
\text { CDI à temps } \\
\text { plein. }\end{array}$ & $\begin{array}{l}\text { Vit avec son } \\
\text { conjoint ( } 30 \\
\text { ans) sans enfant } \\
\text { à charge. }\end{array}$ & $\begin{array}{l}\text { Antécédents } \\
\text { psychiatriques } \\
\text { non } \\
\text { communiqués. } \\
\text { Suivie par un } \\
\text { psychologue } \\
\text { depuis } 1 \text { an. }\end{array}$ & $\begin{array}{l}\text { Fait du sport et } \\
\text { ne se décrit pas } \\
\text { comme active. } \\
\text { A décidé de } \\
\text { recommencer à } \\
\text { faire } \\
\text { régulièrement } \\
\text { de l'activité } \\
\text { physique. }\end{array}$ & $\begin{array}{l}\text { Se décrit comme } \\
\text { quelqu'un } \\
\text { d'accessible à la } \\
\text { spiritualité et } \\
\text { pratique une fois } \\
\text { par an. } \\
\text { AInf }=7 / 7 \text {. } \\
\mathrm{INT}_{\text {diet }}=13 / 14 \text {. } \\
\mathrm{INT}_{\mathrm{AP}}=13 / 14 \text {. } \\
\mathrm{INT}_{\mathrm{psy}}=7 / 7 \text {. }\end{array}$ \\
\hline
\end{tabular}

Légende : IMC : indice de masse corporelle. CSP : catégorie socio-professionnelle. HTA : hypertension artérielle. SAS : syndrome d'apnée du sommeil. DOA : douleurs ostéo-articulaires. DT2 : diabète de type 2 . MCV : maladie cardio- ou cérébro-vasculaire. VIH : virus

d'immunodéficience simienne. PEC : prise en charge en nutrition. TS : tentative de suicide. CMP : centre médico-psychologique. AInf : aise avec l'informatique. $\mathrm{INT}_{\text {diet }}$ : intention de suivre les recommandations diététiques sur les 15 jours suivant l'inclusion. $\mathrm{INT}_{\mathrm{AP}}$ : intention de suivre les recommandations en termes d'activité physique sur les 15 jours suivant l'inclusion. INT $_{\mathrm{psy}}$ : intention de suivre une prise en charge psychothérapeutique expérimentale.

Note: IMC : body mass index. CSP : socioprofessional class. HTA : arterial hypertension. SAS : sleep apnea syndrom. DOA : articular pain. DT2 : type 2 diabetes. MCV : cardiovascular disease. VIH : human immunodeficiency virus. PEC : nutritional care. TS : suicide attempt. CMP :

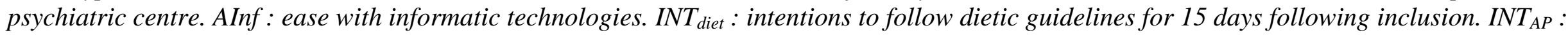
intentions to follow physical activity guidelines for 15 days following inclusion. INT ${ }_{p s y}$ : intentions to comply to an experimental psychotherapy. 
Tableau 2. Statistiques descriptives de la population inclue par condition aux trois premiers temps de mesure.

Table 2. Descriptive statistics of included patients by condition at 0,1 , and 6 months.

\begin{tabular}{|c|c|c|c|c|c|c|c|c|c|}
\hline \multirow[b]{2}{*}{ Variable } & \multicolumn{3}{|c|}{ Inclusion (V0) } & \multicolumn{3}{|c|}{1 mois (V1) } & \multicolumn{3}{|c|}{6 mois (V6) } \\
\hline & GI & GIC & GC & GI & GIC & GC & GI & GIC & GC \\
\hline Poids (kg) & $105,67(3)$ & $108,83(6)$ & $122,67(3)$ & - & - & - & $86,10(1)$ & $123,50(1)$ & NA \\
\hline $\operatorname{IMC}\left(\mathrm{kg} / \mathrm{m}^{2}\right)$ & $36,64(3)$ & $36,05(6)$ & $41,12(3)$ & - & - & - & $34,49(1)$ & $41,75(1)$ & NA \\
\hline Restriction cognitive (TFEQ) & $18,52(3)$ & $48,15(6)$ & $38,89(3)$ & $22,22(1)$ & $50,93(6)$ & $15,71(2)$ & $22,22(1)$ & $16,67(1)$ & $38,89(1)$ \\
\hline Impulsivité alimentaire (TFEQ) & $41,98(3)$ & $68,52(6)$ & $70,37(3)$ & $0,00(1)$ & $54,94(6)$ & $66,67(2)$ & $3,70(1)$ & $25,93(1)$ & $85,19(1)$ \\
\hline Alimentation émotionnelle (TFEQ) & $59,26(3)$ & $87,04(6)$ & $81,48(3)$ & $0,00(1)$ & $74,08(6)$ & $100,00(2)$ & $11,11(1)$ & $66,67(1)$ & $100,00(1)$ \\
\hline Calories consommées $(7 \mathrm{j}$; Kcal) & $2240,23(3)$ & $2105,95(5)$ & $1866,08(3)$ & - & - & - & $1266,91(1)$ & $3777,57(1)$ & NA \\
\hline Pleine conscience (MAAS) & $4,31(3)$ & $2,94(6)$ & $3,42(3)$ & $4,93(1)$ & $2,93(6)$ & $3,20(2)$ & $5,20(1)$ & $3,33(1)$ & $4,07(1)$ \\
\hline Pleine conscience ( $7 \mathrm{j}$; DMRS) & $30,18(2)$ & $16,68(5)$ & $18,93(3)$ & - & - & - & $32,14(1)$ & $8,43(1)$ & $7,57(1)$ \\
\hline Anxiété (HADS) & $8,33(3)$ & $11,50(6)$ & $12,00(3)$ & $8,00(1)$ & $11,33(6)$ & $14,00(2)$ & $7,00(1)$ & $16,00(1)$ & $14,00(1)$ \\
\hline Dépression (HADS) & $4,67(3)$ & $6,67(6)$ & $7,67(3)$ & $10,00(1)$ & $7,33(6)$ & $12,00(2)$ & $9,00(1)$ & $4,00(1)$ & $1,00(1)$ \\
\hline Détresse psychologique (HADS) & $13,00(3)$ & $18,17(6)$ & $19,67(3)$ & $18,00(1)$ & $18,67(6)$ & $26,00(2)$ & $16,00(1)$ & $20,00(1)$ & $15,00(1)$ \\
\hline Amotivation (BREQ) & $0,33(3)$ & $0,00(6)$ & $1,67(3)$ & $0,00(1)$ & $0,38(6)$ & $0,63(2)$ & $0,00(1)$ & $0,00(1)$ & $1,50(1)$ \\
\hline Motivation extrinsèque (BREQ) & $0,42(3)$ & $0,21(6)$ & $0,50(3)$ & $0,00(1)$ & $0,67(6)$ & $0,38(2)$ & $0,00(1)$ & $0,00(1)$ & $3,00(1)$ \\
\hline Motivation intrinsèque (BREQ) & $3,67(3)$ & $3,38(6)$ & $2,33(3)$ & $4,00(1)$ & $3,08(6)$ & $1,50(2)$ & $4,00(1)$ & $4,00(1)$ & $0,00(1)$ \\
\hline Dépense énergétique (IPAQ; METs) & $4973,00(3)$ & $6334,67(6)$ & $11293,00(3)$ & $7812,00(1)$ & $4930,42(6)$ & $1983,44(2)$ & $5130,00(1)$ & $6342,00(1)$ & $13230(1)$ \\
\hline Nombre de pas $(7 \mathrm{j})$ & $7648,22(2)$ & $8085,04(5)$ & $5665,67(3)$ & - & - & - & $4025,29(1)$ & $7094,71(1)$ & $12524,57(1)$ \\
\hline Distance parcourue $(7 \mathrm{j} ; \mathrm{km})$ & $5,52(2)$ & $6,19(4)$ & $4,39(3)$ & - & - & - & $2,89(1)$ & $4,25(1)$ & $8,47(1)$ \\
\hline Temps assis (IPAQ; min) & $270(2)$ & $420(5)$ & $480(2)$ & $180(1)$ & $285(6)$ & $480(2)$ & $60(1)$ & $300(1)$ & $600(1)$ \\
\hline
\end{tabular}

Légende : Les données présentées sont de type "moyenne (nombre de participants)". Les cases vides signifient que la mesure de la variable n'était pas prévue dans le protocole. NA : données manquantes. GI : groupe intervention (pleine conscience). GIC : groupe intervention contrôle (méditation factice). GC : groupe contrôle.

Note: Presented data are "mean (number of participants)". Empty cells stand for unplanned measures at 1 months. NA : missing data. GI : intervention group (mindfulness). GIC : control intervention group (sham meditation). GC : control group. 
Tableau 3. Changements individuels à 1 et 6 mois de l'inclusion.

Table 3. Individual changes at 1 and 6 months after inclusion.

\begin{tabular}{|c|c|c|c|c|c|c|c|c|c|c|}
\hline \multirow[b]{2}{*}{ Variable } & \multirow[b]{2}{*}{ Temps } & \multirow{2}{*}{$\begin{array}{c}\text { GI } \\
\text { P004 }\end{array}$} & \multicolumn{6}{|c|}{ GIC } & \multicolumn{2}{|c|}{ GC } \\
\hline & & & P006 & P007 & P013 & P014 & P015 & P016 & P002 & P003 \\
\hline \multirow[t]{2}{*}{ Restriction cognitive } & 1 mois & 0 & $-5,55$ & $+27,78 *$ & $-16,67$ & $+11,11$ & 0 & 0 & $-27,78 *$ & $-5,56$ \\
\hline & 6 mois & $+16,66$ & $-16,66$ & - & - & - & - & - & - & 0 \\
\hline \multirow[t]{2}{*}{ Impulsivité alimentaire } & 1 mois & $-3,70$ & $-11,11$ & $-25,93 *$ & $-7,41$ & $-3,70$ & $-7,41$ & $-25,93 *$ & $-7,41$ & $-3,70$ \\
\hline & 6 mois & 0 & $-55,55 *$ & - & - & - & - & - & - & $-3,70$ \\
\hline \multirow[t]{2}{*}{ Alimentation émotionnelle } & 1 mois & 0 & 0 & 0 & $-11,11$ & $-22,23 *$ & $-22,22 *$ & $-22,22 *$ & $+22,22 *$ & 0 \\
\hline & 6 mois & $+11,11$ & $-33,33 *$ & - & - & - & - & - & - & 0 \\
\hline Calories consommées & 6 mois & $+258,40$ & $+1092,33$ & - & - & - & - & - & - & - \\
\hline Poids & 6 mois & $-1,90$ & $+0,50$ & - & - & - & - & - & - & - \\
\hline \multirow[t]{2}{*}{ Amotivation } & 1 mois & 0 & 0 & 0 & 0 & $+1,25 *$ & $+1,00$ & 0 & $-2,50 *$ & $-1,25$ \\
\hline & 6 mois & 0 & 0 & - & - & - & - & - & - & $-1,00$ \\
\hline \multirow[t]{2}{*}{ Motivation extrinsèque } & 1 mois & 0 & $+0,75 *$ & 0 & $+0,25$ & 0 & $+2,25 *$ & $-0,50$ & $+0,25$ & 0 \\
\hline & 6 mois & 0 & $-0,25$ & - & - & - & - & - & - & $+2,75 *$ \\
\hline \multirow[t]{2}{*}{ Motivation intrinsèque } & 1 mois & 0 & 0 & 0 & 0 & $-1,00 *$ & $-1,00 *$ & $+0,25$ & $-1,00$ & 0 \\
\hline & 6 mois & 0 & 0 & - & - & - & - & - & - & 0 \\
\hline \multirow[t]{2}{*}{ Dépense énergétique } & 1 mois & +1506 & $-7105,5$ & +495 & -600 & +800 & +6720 & -8735 & $-12678 *$ & $-16983 *$ \\
\hline & 6 mois & -1176 & -6963 & - & - & - & - & - & - & -6678 \\
\hline Temps assis & 6 mois & 0 & +120 & - & - & - & - & - & - & 0 \\
\hline Nombre de pas & 6 mois & $-991,14$ & $-2726,29$ & - & - & - & - & - & - & $+5086,14 *$ \\
\hline Distance parcourue & 6 mois & $-0,47$ & $-2,63$ & - & - & - & - & - & - & $+2,86$ \\
\hline \multirow[t]{2}{*}{ Pleine conscience (MAAS) } & 1 mois & $+0,26$ & $-0,60$ & $+0,40$ & $+0,67$ & $+0,06$ & $-0,67$ & $+0,13$ & $+0,47$ & $-1,33$ \\
\hline & 6 mois & $+0,53$ & $+0,40$ & - & - & - & - & - & - & $-0,26$ \\
\hline Pleine conscience (DMRS) & 6 mois & $-2,72$ & $-0,71$ & - & - & - & - & - & - & $-9,29$ \\
\hline \multirow[t]{2}{*}{ Anxiété } & 1 mois & $+1,00$ & $+3,00$ & $-3,00$ & 0 & $-4,00$ & $+2,00$ & $+1,00$ & $+2,00$ & $+2,00$ \\
\hline & 6 mois & 0 & $+1,00$ & - & - & - & - & - & - & $+3,00$ \\
\hline \multirow[t]{2}{*}{ Dépression } & 1 mois & $+4,00$ & $+4,00$ & $+1,00$ & $-5,00$ & $-1,00$ & $+5,00$ & $-1,00$ & $+9,00 *$ & $+1,00$ \\
\hline & 6 mois & $+3,00$ & $-1,00$ & - & - & - & - & - & - & $-3,00$ \\
\hline \multirow[t]{2}{*}{ Détresse psychologique } & 1 mois & $+5,00$ & $+8,00 *$ & $-2,00$ & $-5,00$ & $-5,00$ & $+7,00$ & 0 & $+11,00 *$ & $+1,00$ \\
\hline & 6 mois & $+3,00$ & 0 & - & - & - & - & - & - & 0 \\
\hline \multirow[t]{2}{*}{ Adhésion (\% fait) } & Phase 1 & 13,33 & 33,33 & 30,00 & 10,00 & 20,00 & - & 60,00 & - & - \\
\hline & Phase 2 & 7,66 & 1,40 & 6,52 & 1,85 & 5,00 & - & 46,15 & - & - \\
\hline
\end{tabular}

* changements cliniquement significatifs. Les cases vides sont des données manquantes.

* clinicaly significant changes. Empty cells stand for missing data. 